\title{
POLICY GRADIENT METHODS FOR THE NOISY LINEAR QUADRATIC REGULATOR OVER A FINITE HORIZON*
}

\author{
BEN HAMBLY ${ }^{\dagger}$, RENYUAN XU ${ }^{\dagger}$, AND HUINING YANG ${ }^{\dagger}$
}

\begin{abstract}
We explore reinforcement learning methods for finding the optimal policy in the linear quadratic regulator (LQR) problem. In particular we consider the convergence of policy gradient methods in the setting of known and unknown parameters. We are able to produce a global linear convergence guarantee for this approach in the setting of finite time horizon and stochastic state dynamics under weak assumptions. The convergence of a projected policy gradient method is also established in order to handle problems with constraints. We illustrate the performance of the algorithm with two examples. The first example is the optimal liquidation of a holding in an asset. We show results for the case where we assume a model for the underlying dynamics and where we apply the method to the data directly. The empirical evidence suggests that the policy gradient method can learn the global optimal solution for a larger class of stochastic systems containing the LQR framework, and that it is more robust with respect to model misspecification when compared to a model-based approach. The second example is an LQR system in a higher dimensional setting with synthetic data.
\end{abstract}

Key words. linear quadratic regulator, reinforcement learning, policy gradient method, stochastic control, optimal liquidation, optimal execution

AMS subject classifications. 68Q25, 68R10, 68U05

DOI. $10.1137 / 20 \mathrm{M} 1382386$

1. Introduction. The linear quadratic regulator (LQR) problem is one of the most fundamental in optimal control theory. Its aim is to find a control for a linear dynamical system; that is, the dynamics of the state of the system is described by a linear function of the current state and input, subject to a quadratic cost. It is an important problem for a number of reasons: (1) the LQR problem is one of the few optimal control problems for which there exists a closed-form analytical representation of the optimal feedback control; (2) when the dynamics are nonlinear and hard to analyze, an LQR approximation may be obtained as a local expansion and provide an approximation that is provably close to the original problem; (3) the LQR has been used in a wide variety of applications. In particular, in the set-up of fixed time horizon and stochastic dynamics, applications include portfolio optimization [3] and optimal liquidation [7] in finance, resource allocation in energy markets [40, 45], and biological movement systems [34].

Until recently much of the work on the LQR problem has focused on solving for the optimal controls under the assumption that the model parameters are fully known. See the book of Anderson and Moore [9] for an introduction to the LQR problem with known parameters. However, assuming that the controller has access to all the model parameters is not realistic for many applications, and this has led to the exploration of learning approaches to the problem. We consider reinforcement learning (RL), one of the three basic machine learning paradigms (alongside supervised learning

* Received by the editors November 24, 2020; accepted for publication (in revised form) June 15, 2021; published electronically September 28, 2021.

https://doi.org/10.1137/20M1382386

Funding: The third author was supported by the EPSRC Centre for Doctoral Training in Industrially Focused Mathematical Modelling (EP/L015803/1) in collaboration with BP plc.

$\dagger$ Mathematical Institute, University of Oxford, Oxford, OX2 6GG, UK (hambly@maths.ox.ac.uk, xur@maths.ox.ac.uk, yang@maths.ox.ac.uk). 
and unsupervised learning). Unlike the situation with full information on the model parameters, RL is learning to make decisions via trial and error, through interactions with the (partially) unknown environment. In RL, an agent takes an action and receives a reinforcement signal in terms of a numerical reward, which encodes the outcome of her action. In order to maximize the accumulated reward over time, the agent learns to select her actions based on her past experiences (exploitation) and/or by making new choices (exploration). There are two popular approaches in RL to handle the LQR with unknown parameters: the model-based approach and the model-free approach.

In the paradigm of the model-based approach, the controller estimates the unknown model parameters and then constructs a control policy based on the estimated parameters. The classical approach is the certainty equivalence principle [10]: the unknown parameters are estimated using observations (or samples), and a control policy is then designed by treating the estimated parameters as the truth. In the first step, the unknown model parameters can be estimated by standard statistical methods such as least-squares minimization [19]. The second step is to show that when the estimated parameters are accurate enough, the policy using the "plug-in" estimates enjoys good theoretical guarantees of being close to optimal. See [19] and [23] for the optimal gap and sample complexities along this line, and see [21] for the sample complexity with distributed robust learning. Another line of work in the model-based regime focuses on uncertainty quantification. The controller updates her posterior belief or the confidence bounds on the unknown model parameters and then makes decisions in an online manner; see [1, 2, 20, 31, 39].

Another recently developed approach is the model-free approach, where the controller learns the optimal policy directly via interacting with the system, without inferring the model parameters. As the optimal policy in the LQR problem is a linear function of the state, the aim is to determine this linear function. This is equivalent to learning a set of parameters in matrix form, called the policy matrix. One natural way to achieve this goal is to apply the gradient descent method in the parameter space of the policy matrix, also referred to as the policy gradient method. In particular, the policy gradient method computes the gradient of the cost function with respect to the policy matrix and then updates the policy in the steepest decent direction to find the optimal policy. The paper [22] was the first to show that policy gradients converge to the global optimal solution with polynomial (in the relevant quantities) sample complexity. However, [22] focuses on the case where the only noise in the system is in the initial state, and the rest of the state transitions are deterministic. There are other methods that fall into the category of the model-free approach, including the actor-critic method [46] and least-squares temporal difference learning [43].

If the true system is indeed linear quadratic, the model-based approaches (may) outperform the model-free approaches by fully utilizing the linear quadratic structure. For example, in the setting where the system transition matrices are unknown and the parameters in the cost function are known, [42] and [44] showed that model-based methods are (asymptotically) more sample-efficient than some popular model-free methods. However, we are often uncertain about whether the actual system is linear quadratic in the learning setting; for instance, there might be some small nonlinear terms in the system dynamics. Therefore, compared to the model-based approach, which strongly relies on the assumption that the stochastic system lies within the LQR framework and may, in practice, suffer from model misspecification, the execution of the model-free algorithm does not rely on the assumptions of the model. It has been shown that the policy gradient method can learn the global optimal solution, not only 
for the LQR framework, but also for a more general class of deterministic systems in the setting of an infinite time horizon [13]. Thus the advantage of the model-free approach is that it is more robust against model misspecification compared to the model-based approach.

Our contributions. We now summarize our contributions. Motivated by many real-word decision-making problems with a fixed deadline and uncertainty in the underlying dynamics, such as the optimal liquidation problem that we discuss in section 2, we extend the framework of [22] by incorporating a finite time horizon and sub-Gaussian noise (which includes Gaussian noise as a special case). In particular, we provide a global linear convergence guarantee and a polynomial sample complexity guarantee for the policy gradient method in this setting with both known parameters (Theorem 3.3) and unknown parameters (Theorem 4.4). The analysis with known parameters paves the way for learning LQR with unknown parameters. In addition, numerically solving the Riccati equation with known parameters in high dimensions may suffer from computational inaccuracy. The policy gradient method provides a direct way of searching for the optimal solution with known parameters in this case, which may be of separate interest. Note that the optimal policy is time-invariant for the LQR with infinite time horizon, whereas the optimal policy is time-dependent with finite time horizon and hence harder to learn in general. With noise in the dynamics, we need more careful choices of the hyperparameters to retrieve compatible sample complexities with noisy observations. In addition, when optimal polices need to satisfy certain constraints, we provide a global convergence result for the projected policy gradient method in Theorem 4.5. This is required in the context of our application to the optimal liquidation problem.

We will formulate the optimal liquidation problem over a fixed horizon as a noisy LQR problem which is essentially the classical Almgren-Chriss formulation [7]. The performance of the algorithm on NASDAQ ITCH data is assessed. As well as using the method within this modelling approach, we also consider the performance of the policy gradient method when applied directly to the data with an appropriate cost function. This improves the performance of the LQR/Almgren-Chriss solution and shows promising results for the use of the policy gradient method for problems that are "close" to the LQR framework.

\subsection{Related work.}

Policy gradient methods for $L Q R$ problems. Since the policy gradient method is the main focus of our paper, here we provide a review of the previous theoretical work on this method in various LQR settings and extensions. The first global convergence result for the policy gradient method to learn the optimal policy for LQR problems was developed in [22] in the setting of infinite horizon and deterministic dynamics. The work of [22] was extended in [13] to give global optimality guarantees of policy gradient methods for a larger class of control problems that includes the linear quadratic case. In particular, this class of control problems satisfies a closure condition under policy improvement and convexity of policy improvement steps. The paper [14] considers policy gradient methods for LQR problems in terms of optimizing a real valued matrix function over the set of feedback gains. The extension of the policy gradient method to continuous-time can be found in [15]. All of these methods are in the infinite horizon setting and without the addition of noise in the dynamics.

There has been some work on the case of noisy dynamics, but all in the setting of infinite horizon. In [26] the problem with a multiplicative noise was discussed, using a relatively straightforward extension of the deterministic dynamics considered in the 
original framework. In the case of additive noise, [32] studies the global convergence of policy gradient and other learning algorithms for the LQR over an infinite time horizon and with Gaussian noise. In particular, the policy considered in [32] is a randomized policy with Gaussian distribution. There is also [35], which studies derivative-free (zeroth-order) policy optimization methods for the LQR with bounded additive noise. Finally some other contributions can be found in $[16,47]$ for zero-sum LQR games and in $[17,28]$ for mean-field LQR games.

Compared to [22], our technical difficulties are three-fold. First, due to the timedependent nature of the admissible policies over a finite horizon and randomness from the system noise, we need additional conditions and analysis to guarantee the well-definedness of the state process, i.e., the nondegeneracy of the controlled statecovariance matrices. This holds almost for free in the infinite horizon case with deterministic dynamics. Second, we need to take care of the additional randomness from the sub-Gaussian noise when developing the perturbation analysis and the gradient dominant condition. Third, we need more advanced concentration inequalities and tighter upper bounds to provide compatible sample complexity analysis in the unknown parameter case. See the more detailed discussion in Remark 4.12.

Optimal liquidation. An early mathematical framework for the optimal liquidation problem is due to Almgren and Chriss [7]. In this problem a trader is required to liquidate a portfolio of shares over a fixed horizon. The selling of a large number of shares at once has both temporary and permanent impacts on the share price causing it to decrease. The trader therefore wishes to find a trading strategy which maximizes her return from, or alternatively, minimizes the cost of, the liquidation of the portfolio subject to a given level of risk.

This problem has been considered in many papers and extended in many directions. See, for instance, $[5,6,25]$. We will cast this as an LQR problem and show how the policy gradient method is a powerful tool for solving this problem even without assumptions on the model.

More recently techniques from reinforcement learning have been applied to the optimal liquidation problem. The first paper to do this was [37], where the authors showed promising results for this approach by designing a Q-learning-based algorithm to optimally select price levels and passively place limit orders. This was further developed in [30], which designed a Q-learning-based algorithm for liquidation within the standard Almgren-Chriss framework. For recent work incorporating deep learning see, for example, $[11,33,38,48]$. See [18] and the references therein for a detailed review on reinforcement learning with applications in finance and economics. However, all these works focus on the model-free setting without taking advantage of even weak modelling assumptions on the market dynamics. In addition, the performances of these proposed algorithms are validated only through empirical studies and no theoretical guarantee of convergence is provided.

Organization and notation. For any matrix $Z=\left(Z_{1}, \ldots, Z_{d}\right) \in \mathbb{R}^{m \times d}$ with $Z_{j} \in$ $\mathbb{R}^{m}(j=1,2, \ldots, d), Z^{\top} \in \mathbb{R}^{m \times d}$ denotes the transpose of $Z ;\|Z\|$ denotes the spectral norm of a matrix $Z ; \operatorname{Tr}(Z)$ denotes the trace of a square matrix $Z ;\|Z\|_{F}$ denotes the Frobenius norm of a matrix $Z ; \sigma_{\min }(Z)$ denotes the minimal singular value of a square matrix $Z$; and $\operatorname{vec}(Z)=\left(Z_{1}^{\top}, \ldots, Z_{d}^{\top}\right)^{\top}$ denotes the vectorized version of a matrix $Z$. For a sequence of matrices $\boldsymbol{D}=\left(D_{0}, \ldots, D_{T}\right)$, we define a new norm $\|\boldsymbol{D}\|$ as $\|\boldsymbol{D}\|=\sum_{t=0}^{T}\left\|D_{t}\right\|$, where $D_{t} \in \mathbb{R}^{m \times d}$. We further denote $\mathcal{N}(\mu, \Sigma)$ as the Gaussian distribution with mean $\mu \in \mathbb{R}^{d}$ and covariance matrix $\Sigma \in \mathbb{R}^{d \times d}$.

The rest of the paper is organized as follows. We introduce the mathematical 
framework and problem set-up in section 2. The first step in our convergence analysis of the policy gradient method is to consider the case of known model parameters in section 3. When parameters are unknown, the convergence results for the samplebased policy gradient method and projected policy gradient method are obtained in section 4. Finally, the algorithm is applied to a liquidation problem. See sections 2.1 and 5 for the corresponding set-up and algorithm performance, respectively.

We provide some technical proofs in the longer arXiv version of our paper [29], which can be found at https://arxiv.org/pdf/2011.10300.pdf.

2. Problem set-up. We consider the following LQR problem over a finite time horizon $T$ :

$$
\min _{\left\{u_{t}\right\}_{t=0}^{T-1}} \mathbb{E}\left[\sum_{t=0}^{T-1}\left(x_{t}^{\top} Q_{t} x_{t}+u_{t}^{\top} R_{t} u_{t}\right)+x_{T}^{\top} Q_{T} x_{T}\right],
$$

such that for $t=0,1, \ldots, T-1$,

$$
x_{t+1}=A x_{t}+B u_{t}+w_{t}, x_{0} \sim \mathcal{D} .
$$

Here $x_{t} \in \mathbb{R}^{d}$ is the state of the system with the initial state $x_{0}$ drawn from a distribution $\mathcal{D}, u_{t} \in \mathbb{R}^{k}$ is the control at time $t$, and $\left\{w_{t}\right\}_{t=0}^{T-1}$ are zero-mean independent and identically distributed (i.i.d.) noises which are independent from $x_{0}$. At this moment, we only assume $x_{0}$ and $\left\{w_{t}\right\}_{t=0}^{T-1}$ have finite second moments. That is, $\mathbb{E}\left[x_{0} x_{0}^{\top}\right]$ and $W:=\mathbb{E}\left[w_{t} w_{t}^{\top}\right](\forall t=0,1, \ldots, T-1)$ exist. The system parameters $A \in \mathbb{R}^{d \times d}$ and $B \in \mathbb{R}^{d \times k}$ are referred to as system (transition) matrices; $Q_{t} \in \mathbb{R}^{d \times d}(\forall t=0,1, \ldots, T)$ and $R_{t} \in \mathbb{R}^{k \times k}(\forall t=0,1, \ldots, T-1)$ are matrices that parameterize the quadratic costs. Note that the expectation in (2.1) is taken with respect to both $x_{0} \sim \mathcal{D}$ and $w_{t}(t=0,1, \ldots, T-1)$. We further denote by $\boldsymbol{u}:=\left(u_{0}, \ldots, u_{T-1}\right), \boldsymbol{x}:=\left(x_{0}, \ldots, x_{T}\right)$, $\boldsymbol{w}:=\left(w_{0}, \ldots, w_{T-1}\right), \boldsymbol{Q}:=\left(Q_{0}, \ldots, Q_{T}\right)$, and $\boldsymbol{R}:=\left(R_{0}, \ldots, R_{T-1}\right)$ the profile over the decision period $T$.

To solve the LQR problem (2.1)-(2.2), let us start with some conditions on the model parameters to assure the well-definedness of the problem.

Assumption 2.1 (cost parameter). Assume that $Q_{t} \in \mathbb{R}^{d \times d}$ for $t=0,1, \ldots, T$ and $R_{t} \in \mathbb{R}^{k \times k}$ for $t=0,1, \ldots, T-1$ are positive definite matrices.

Under Assumption 2.1, we can properly define a sequence of matrices $\left\{P_{t}^{*}\right\}_{t=0}^{T}$ as the solution to the following dynamic Riccati equation [12]:

$$
P_{t}^{*}=Q_{t}+A^{\top} P_{t+1}^{*} A-A^{\top} P_{t+1}^{*} B\left(B^{\top} P_{t+1}^{*} B+R_{t}\right)^{-1} B^{\top} P_{t+1}^{*} A,
$$

with terminal condition $P_{T}^{*}=Q_{T}$. The matrices $\left\{P_{t}^{*}\right\}_{t=0}^{T}$ can be found by solving the Riccati equations iteratively backwards in time. In particular with a slight modification of the initial state distribution in [12, Chapter 4.1], we have the following result.

Lemma 2.2 (well-definedness and the optimal solution [12]). Under Assumption 2.1, the following hold:

1. The solution $P_{t}^{*}$ to the Riccati equation (2.3) is positive definite $\forall t=0,1, \ldots, T$.

2. Then the optimal control sequence $\left\{u_{t}\right\}_{t=0}^{T-1}$ is given by

$$
\begin{aligned}
u_{t} & =-K_{t}^{*} x_{t}, \quad \text { where } \\
K_{t}^{*} & =\left(B^{\top} P_{t+1}^{*} B+R_{t}\right)^{-1} B^{\top} P_{t+1}^{*} A .
\end{aligned}
$$


To find the optimal solution in the linear feedback form (2.4), we only need to focus on the following class of linear admissible policies in feedback form:

$$
u_{t}=-K_{t} x_{t}, \quad t=0,1, \ldots, T-1,
$$

which can be fully characterized by $K:=\left(K_{0}, \ldots, K_{T-1}\right)$.

2.1. Application: The optimal liquidation problem. One application of the LQR framework (2.1)-(2.2) is the optimal liquidation problem. We give a slight variant of the setup of Almgren and Chriss [7]. Our aim is to liquidate an amount $q_{0}$ of an asset, with price $S_{0}$ at time 0 , over the time period $[0, T]$ with trading decisions made at discrete time points $t=0,1, \ldots, T-1$. At each time $t$ our decision is to liquidate an amount $u_{t}$ of the asset. Any residual holding is then liquidated at time $T$. This will have two types of price impact. There will be a temporary price impact, caused when the order "walks the book," and a permanent price impact as traders rearrange their positions in light of the sell order. We will assume the impacts are linear in the number of traded shares.

We write $S_{t}$ for the asset price at time $t$. This evolves according to a Bachelier model with a linear permanent price impact in that

$$
S_{t+1}=S_{t}+\sigma Z_{t+1}-\gamma u_{t},
$$

where, for each $t=1, \ldots, T, Z_{t}$ is an independent standard normal random variable, $\sigma$ is the volatility, and $\gamma$ is the permanent price impact parameter. The inventory process $q_{t}$ records the current holding in the asset at time $t$. Thus we have

$$
q_{t+1}=q_{t}-u_{t} .
$$

Therefore, the two-dimensional state process is

$$
\left(\begin{array}{c}
S_{t+1} \\
q_{t+1}
\end{array}\right)=\left(\begin{array}{ll}
1 & 0 \\
0 & 1
\end{array}\right)\left(\begin{array}{c}
S_{t} \\
q_{t}
\end{array}\right)+\left(\begin{array}{l}
-\gamma \\
-1
\end{array}\right) u_{t}+\left(\begin{array}{c}
\sigma Z_{t+1} \\
0
\end{array}\right)
$$

When selling shares we incur a temporary price impact, parameter $\beta$, in that if, at time $t$, we trade $u_{t}$ of our asset, then we obtain $\tilde{S}_{t}=S_{t}-\beta u_{t}$ per share. Therefore the total revenue is $\sum_{t=0}^{T-1} u_{t} \tilde{S}_{t}+q_{T} \tilde{S}_{T}$, and $C_{T}$, the total cost of execution over $[0, T]$, is the book value at time 0 minus the revenue:

$$
C_{T}=q_{0} S_{0}-\sum_{t=0}^{T-1} u_{t} \tilde{S}_{t}-q_{T} \tilde{S}_{T} .
$$

In a way similar to [7], after summation by parts, we have

$$
C_{T}=-\sigma \sum_{t=1}^{T} q_{t} Z_{t}-\frac{\gamma}{2} \sum_{t=0}^{T-1} u_{t}^{2}+\frac{\gamma}{2}\left(q_{0}^{2}-q_{T}^{2}\right)+\beta \sum_{t=0}^{T-1} u_{t}^{2}+\beta q_{T}^{2} .
$$

The mean and variance of the total cost of execution are given by

$$
\mathbb{E}(C)=\sum_{t=0}^{T-1} \delta u_{t}^{2}+\delta q_{T}^{2}+\frac{\gamma}{2} q_{0}^{2}, \quad \operatorname{var}(C)=\sum_{t=1}^{T} \sigma^{2} q_{t}^{2},
$$

where $\delta=\beta-\gamma / 2$ summarizes the impact and is assumed positive. 
Following Almgren and Chriss [7], we minimize the following cost function:

$$
C_{\mathrm{AC}}=\min (\mathbb{E}(C)+\phi \operatorname{var}(C)),
$$

where $\phi$ is a parameter balancing risk versus return. For our LQR framework we take the cost function to be

$$
\begin{aligned}
C_{\mathrm{LQR}}(\epsilon) & =\min \left(\mathbb{E}(C)+\phi \operatorname{var}(C)+\epsilon \sum_{t=0}^{T} S_{t}^{2}\right) \\
& =\min \left(\sum_{t=0}^{T-1} \delta u_{t}^{2}+\delta q_{T}^{2}+\frac{\gamma}{2} q_{0}^{2}+\phi \sum_{t=1}^{T} \sigma^{2} q_{t}^{2}+\epsilon \sum_{t=0}^{T} S_{t}^{2}\right) .
\end{aligned}
$$

Note that the term $\epsilon \sum_{t=0}^{T} S_{t}^{2}$, with some small $\epsilon>0$, serves as a regularization term to guarantee Assumption 2.1 holds. In practice, we can show that the optimal solution with $\epsilon$ small is close to the Almgren-Chriss solution (when $\epsilon=0$ ). In addition, the algorithm will still converge with $\epsilon=0$. See further discussion in section 5 . Thus, in the LQR formulation we have

$$
A=\left(\begin{array}{ll}
1 & 0 \\
0 & 1
\end{array}\right), \quad B=(-\gamma,-1)^{\top}, \quad \text { and } \quad w_{t}=\left(\sigma Z_{t+1}, 0\right)^{\top}
$$

and the objective function has

$$
Q_{T}=\left(\begin{array}{cc}
\epsilon & 0 \\
0 & \delta+\phi \sigma^{2}
\end{array}\right), \quad Q_{t}=\left(\begin{array}{cc}
\epsilon & 0 \\
0 & \phi \sigma^{2}
\end{array}\right), \quad \text { and } \quad R_{t}=\delta .
$$

It is easy to see that $Q_{t}$ for $t=0,1, \ldots, T$ and $R_{t}$ for $t=0,1, \ldots, T-1$ are positive definite; hence Assumption 2.1 is satisfied.

We will show that the problem is well-defined and can be solved using the methods of this paper with rigorous convergence guarantees.

3. Exact gradient methods with known parameters. In this section we assume all the parameters in the model, $\left\{Q_{t}\right\}_{t=0}^{T},\left\{R_{t}\right\}_{t=0}^{T-1}, A, B$, are known. The analysis of exact gradient methods with known parameters paves the way for learning LQR with unknown parameters in section 4 . In addition, the policy gradient method provides an alternative way to solve the LQR problem when the parameters are fully known. In this setting the Riccati equation (2.3) is just solved backward in time. However, this operation involves inverting large matrices when the problem is in high dimensions, which may lead to high computational cost and accumulation of computational errors.

Since an admissible policy can be fully characterized by $\boldsymbol{K}$, the cost of a policy $K$ can be correspondingly defined as

$$
C(\boldsymbol{K})=\mathbb{E}\left[\sum_{t=0}^{T-1}\left(x_{t}^{\top} Q_{t} x_{t}+u_{t}^{\top} R_{t} u_{t}\right)+x_{T}^{\top} Q_{T} x_{T}\right],
$$

where $\left\{x_{t}\right\}_{t=1}^{T}$ and $\left\{u_{t}\right\}_{t=0}^{T-1}$ are the dynamics and controls induced by following $\boldsymbol{K}$, starting with $x_{0} \sim \mathcal{D}$. Recall that $\boldsymbol{K}^{*}$ is the optimal policy for the problem, in that

$$
\boldsymbol{K}^{*}=\underset{\boldsymbol{K}}{\arg \min } C(\boldsymbol{K}),
$$

subject to the dynamics (2.2). 
Well-definedness of the state process. To prove the global convergence of policy gradient methods, the essential idea is to show the gradient dominance condition, which states that $C(\boldsymbol{K})-C\left(\boldsymbol{K}^{*}\right)$ can be bounded by $\|\nabla C(\boldsymbol{K})\|_{F}$ for any admissible policy $\boldsymbol{K}$. One of the key steps to guarantee this gradient dominance condition is the well-definedness of the state covariance matrix. That is, $\mathbb{E}\left[x_{t} x_{t}^{\top}\right]$ is positive definite for $t=0,1, \ldots, T$. This condition holds almost for free for LQR problems with infinite time horizon and deterministic dynamics. The only condition needed there is the positive definiteness of $\mathbb{E}\left[x_{0} x_{0}^{\top}\right]$ (see [22]). However, some effort needs to be made to ensure that the state covariance matrix is well-defined for LQR problems with finite horizon and stochastic dynamics. We show that this condition holds under moderate conditions.

Assumption 3.1 (initial state and noise process (I)). We assume that the following hold:

1. Initial state: $x_{0} \sim \mathcal{D}$ such that $\mathbb{E}\left[x_{0} x_{0}^{\top}\right]$ is positive definite.

2. Noise: $\left\{w_{t}\right\}_{t=0}^{T-1}$ are i.i.d. and independent from $x_{0}$ such that $\mathbb{E}\left[w_{t}\right]=0$, and $W=\mathbb{E}\left[w_{t} w_{t}^{\top}\right]$ is positive definite $\forall t=0,1, \ldots, T-1$.

Define $\underline{\sigma}_{\boldsymbol{X}}$ as the lower bound over all the minimum singular values of $\mathbb{E}\left[x_{t} x_{t}^{\top}\right]$ :

$$
\underline{\sigma}_{\boldsymbol{X}}=\min _{t} \sigma_{\min }\left(\mathbb{E}\left[x_{t} x_{t}^{\top}\right]\right)
$$

then we have the following result, and the proof can be found in [29].

LEMma 3.2 (well-definedness of the state covariance matrix). Under Assumption 3.1 , we have that $\mathbb{E}\left[x_{t} x_{t}^{\top}\right]$ is positive definite for $t=0,1, \ldots, T$ under any control policy $\boldsymbol{K}$. Therefore, $\underline{\sigma}_{\boldsymbol{X}}>0$.

Lemma 3.2 implies that if the initial state and the noise driving the dynamics are nondegenerate, the covariance matrices of the state dynamics are positive definite for any policy $\boldsymbol{K}$. However, the covariance matrix may be degenerate in many applications, especially when inventory processes are involved. (See, for example, the liquidation problem (2.7).) In this case, some problem-dependent conditions are needed to guarantee that $\underline{\sigma}_{\boldsymbol{X}}>0$ holds. See further discussion on the condition $\underline{\sigma}_{\boldsymbol{X}}>0$ for the liquidation problem in section 5.1. In light of this we will assume that $\underline{\sigma}_{\boldsymbol{X}}>0$ in the analysis of the convergence of the algorithm in sections 3 and 4 .

Similarly, we define $\underline{\sigma}_{\boldsymbol{R}}$ and $\underline{\sigma}_{\boldsymbol{Q}}$ to be the smallest values of all the minimum singular values of $\boldsymbol{R}$ and $\boldsymbol{Q}$ :

$$
\begin{aligned}
& \underline{\sigma}_{\boldsymbol{R}}=\min _{t} \sigma_{\min }\left(R_{t}\right), \\
& \underline{\sigma}_{Q}=\min _{t} \sigma_{\min }\left(Q_{t}\right) .
\end{aligned}
$$

Under Assumption 2.1, we have $\underline{\sigma}_{\boldsymbol{R}}>0$ and $\underline{\sigma}_{Q}>0$.

We write $\mathcal{H}=\{h \mid h$ are polynomials in the model parameters $\}$ and $\mathcal{H}($.$) when$ there are other dependencies. The model parameters are in terms of $d, k, \frac{1}{\|A\|}, \frac{1}{\|A\|+1}$, $\|A\|, \frac{1}{\|B\|}, \frac{1}{\|B\|+1},\|B\|, \frac{1}{\|R\|}, \frac{1}{\|R\|+1},\|R\|, \frac{1}{\|W\|}, \frac{1}{\|W\|+1},\|W\|, \frac{1}{\sigma_{Q}}, \frac{1}{\sigma_{Q}+1}, \underline{\sigma}_{Q}, \frac{1}{\underline{\sigma}_{\boldsymbol{R}}}, \frac{1}{\underline{\sigma}_{\boldsymbol{R}}+1}$, $\underline{\sigma}_{\boldsymbol{R}}, \frac{1}{\underline{\sigma}_{\boldsymbol{X}}}, \frac{1}{\underline{\sigma}_{\boldsymbol{X}}+1}, \underline{\sigma}_{\boldsymbol{X}},\|\boldsymbol{Q}\|, \mathbb{E}\left[x_{0} x_{0}^{\top}\right]$, and $\frac{1}{\mathbb{E}\left[x_{0} x_{0}^{\top}\right]}$.

Exact gradient descent. We consider the following exact gradient descent updating rule to find the optimal solution (3.2):

$$
K_{t}^{n+1}=K_{t}^{n}-\eta \nabla_{t} C\left(\boldsymbol{K}^{n}\right) \quad \forall 0 \leq t \leq T-1,
$$


where $n$ is the number of iterations, $\nabla_{t} C(\boldsymbol{K})=\frac{\partial C(\boldsymbol{K})}{\partial K_{t}}$ is the gradient of $C(\boldsymbol{K})$ with respect to $K_{t}$, and $\eta$ is the step size. We further denote $\nabla C(\boldsymbol{K})=\left(\nabla_{0} C(\boldsymbol{K}), \ldots\right.$, $\left.\nabla_{T-1} C(\boldsymbol{K})\right)$.

Let us define the state covariance matrix

$$
\Sigma_{t}=\mathbb{E}\left[x_{t} x_{t}^{\top}\right], t=0,1, \ldots, T,
$$

where $\left\{x_{t}\right\}_{t=1}^{T}$ is a state trajectory generated by $\boldsymbol{K}$. Further define a matrix $\Sigma_{\boldsymbol{K}}$ as the sum of $\Sigma_{t}$,

$$
\Sigma_{\boldsymbol{K}}=\sum_{t=0}^{T} \Sigma_{t}=\mathbb{E}\left[\sum_{t=0}^{T} x_{t} x_{t}^{\top}\right] .
$$

Then, the main result for this setting is the following.

TheOREm 3.3 (global convergence of gradient methods). Assume Assumption 2.1 holds. Further assume that $\underline{\sigma}_{\boldsymbol{X}}>0$ and $C\left(\boldsymbol{K}^{0}\right)$ is finite. Then, for an appropriate (constant) setting of the step size $\eta \in \mathcal{H}\left(\frac{1}{C\left(\boldsymbol{K}^{0}\right)+1}\right)$, and for $\epsilon>0$, if we have

$$
N \geq \frac{\left\|\Sigma_{\boldsymbol{K}^{*}}\right\|}{2 \eta \underline{\sigma}_{\boldsymbol{X}}^{2} \underline{\sigma}_{\boldsymbol{R}}} \log \frac{C\left(\boldsymbol{K}^{0}\right)-C\left(\boldsymbol{K}^{*}\right)}{\epsilon},
$$

the exact gradient descent method (3.6) enjoys the following performance bound:

$$
C\left(\boldsymbol{K}^{N}\right)-C\left(\boldsymbol{K}^{*}\right) \leq \epsilon .
$$

The proof of Theorem 3.3 relies on the regularity of the LQR problem, some properties of the gradient descent dynamics, and the perturbation analysis of the covariance matrix of the controlled dynamics.

3.1. Regularity of the LQR problem and properties of the gradient descent dynamics. Let us start with the analysis of some properties of the LQR problem (2.1)-(2.2). To start, Proposition 3.4 focuses on the well-definedness of the Ricatti system $\left\{P_{t}^{K}\right\}_{t=0}^{T}$ induced by a control $\boldsymbol{K}$; Lemma 3.5 gives a representation of the gradient term; Lemma 3.6 and Lemma 3.7 provide the gradient dominance condition and a smoothness condition on the cost function $C(\boldsymbol{K})$ with respect to policy $\boldsymbol{K}$, respectively; and finally, Lemma 3.8 gives two useful upper bounds on Ricatti system and state covariance matrices.

In the finite time horizon setting, define $P_{t}^{K}$ as the solution to

$$
P_{t}^{K}=Q_{t}+K_{t}^{\top} R_{t} K_{t}+\left(A-B K_{t}\right)^{\top} P_{t+1}^{K}\left(A-B K_{t}\right), \quad t=0,1, \ldots, T-1,
$$

with terminal condition

$$
P_{T}^{K}=Q_{T}
$$

Note that (3.9) is equivalent to the Riccati equation (2.3) with optimal $K_{t}=K_{t}^{*}$ as given by (2.5). We have the following result on the well-definedness of $P_{t}^{K}$, the proof of which can be found in [29].

Proposition 3.4. Under Assumption 2.1, the matrices $P_{t}^{K}$ for $t=0,1, \ldots, T$ derived from (3.9) are positive definite.

To ease the exposition, we write $P_{t}^{K}$ as $P_{t}$ when there is no confusion. Then the cost of $K$ can be rewritten as

$$
C(\boldsymbol{K})=\mathbb{E}_{x_{0} \sim \mathcal{D}}\left[x_{0}^{\top} P_{0} x_{0}+L_{0}\right],
$$

Copyright $@$ by SIAM. Unauthorized reproduction of this article is prohibited. 
where, for $t=0,1, \ldots, T-1$,

$$
L_{t}=L_{t+1}+\mathbb{E}\left[w_{t}^{\top} P_{t+1} w_{t}\right]=L_{t+1}+\operatorname{Tr}\left(W P_{t+1}\right),
$$

with $L_{T}=0$. To see this,

$$
\begin{aligned}
\mathbb{E} & \left.x_{0}^{\top} P_{0} x_{0}\right]+L_{0} \\
& =\mathbb{E}\left[x_{0}^{\top} Q_{0} x_{0}+x_{0}^{\top} K_{0}^{\top} R_{0} K_{0} x_{0}+x_{0}^{\top}\left(A-B K_{0}\right)^{\top} P_{1}\left(A-B K_{0}\right) x_{0}+\sum_{t=0}^{T-1} w_{t}^{\top} P_{t+1} w_{t}\right] \\
& =\mathbb{E}\left[x_{0}^{\top} Q_{0} x_{0}+u_{0}^{\top} R_{0} u_{0}+x_{1}^{\top} P_{1} x_{1}+\sum_{t=1}^{T-1} w_{t}^{\top} P_{t+1} w_{t}\right] \\
& =\mathbb{E}\left[\sum_{t=0}^{T-1}\left(x_{t}^{\top} Q_{t} x_{t}+u_{t}^{\top} R_{t} u_{t}\right)+x_{T}^{\top} Q_{T} x_{T}\right] .
\end{aligned}
$$

In addition, define

$$
E_{t}=\left(R_{t}+B^{\top} P_{t+1} B\right) K_{t}-B^{\top} P_{t+1} A, \quad t=0,1, \ldots, T-1 .
$$

Then we have the following representation of the gradient term.

LEMma 3.5. The policy gradient has the following form for $t=0,1, \ldots, T-1$ :

$$
\nabla_{t} C(\boldsymbol{K})=2\left(\left(R_{t}+B^{\top} P_{t+1} B\right) K_{t}-B^{\top} P_{t+1} A\right) \mathbb{E}\left[x_{t} x_{t}^{\top}\right]=2 E_{t} \Sigma_{t}
$$

Proof. Since

$$
\begin{aligned}
C(\boldsymbol{K}) & =\mathbb{E}\left[x_{0}^{\top} P_{0} x_{0}+L_{0}\right] \\
& =\mathbb{E}\left[x_{0}^{\top}\left(Q_{0}+K_{0}^{\top} R_{0} K_{0}\right) x_{0}+x_{0}^{\top}\left(A-B K_{0}\right)^{\top} P_{1}\left(A-B K_{0}\right) x_{0}+\sum_{t=0}^{T-1} w_{t}^{\top} P_{t+1} w_{t}\right],
\end{aligned}
$$

we have

$\nabla_{0} C(\boldsymbol{K})=\frac{\partial C(\boldsymbol{K})}{\partial K_{0}}=\mathbb{E}\left[2 R_{0} K_{0} x_{0} x_{0}^{\top}-2 B^{\top} P_{1}\left(A-B K_{0}\right) x_{0} x_{0}^{\top}\right]=2 E_{0} \mathbb{E}\left[x_{0} x_{0}^{\top}\right]=2 E_{0} \Sigma_{0}$.

Similarly, $\forall t=0,1, \ldots, T-1$,

$$
\nabla_{t} C(\boldsymbol{K})=2\left(\left(R_{t}+B^{\top} P_{t+1} B\right) K_{t}-B^{\top} P_{t+1} A\right) \mathbb{E}\left[x_{t} x_{t}^{\top}\right]=2 E_{t} \mathbb{E}\left[x_{t} x_{t}^{\top}\right]=2 E_{t} \Sigma_{t},
$$

where the expectation $\mathbb{E}$ is taken with respect to both initial distribution $x_{0} \sim \mathcal{D}$ and noises $\boldsymbol{w}$.

In classical optimization theory [22], gradient domination and smoothness of the objective function are two key conditions to guarantee the global convergence of the gradient descent methods. To prove that $C(\boldsymbol{K})$ is gradient dominated, we first prove Lemma 3.6, which indicates that for a policy $\boldsymbol{K}$, the distance between $C(\boldsymbol{K})$ and the optimal cost $C\left(\boldsymbol{K}^{*}\right)$ is bounded by the sum of the magnitude of the gradient $\nabla_{t} C(\boldsymbol{K})$ for $t=0,1, \ldots, T-1$. 
Lemma 3.6. Assume Assumption 2.1 and $\underline{\sigma}_{\boldsymbol{X}}>0$. Let $\boldsymbol{K}^{*}$ be an optimal policy and $C(\boldsymbol{K})$ be finite; then

$$
\begin{aligned}
\underline{\sigma}_{\boldsymbol{X}} \sum_{t=0}^{T-1} \frac{1}{\left\|R_{t}+B^{\top} P_{t+1} B\right\|} \operatorname{Tr}\left(E_{t}^{\top} E_{t}\right) & \leq C(\boldsymbol{K})-C\left(\boldsymbol{K}^{*}\right) \\
& \leq \frac{\left\|\Sigma_{\boldsymbol{K}^{*}}\right\|}{4 \underline{\sigma}^{2} \underline{\sigma}_{\boldsymbol{R}}} \sum_{t=0}^{T-1} \operatorname{Tr}\left(\nabla_{t} C(\boldsymbol{K})^{\top} \nabla_{t} C(\boldsymbol{K})\right),
\end{aligned}
$$

where $\underline{\sigma}_{\boldsymbol{X}}$ and $\underline{\sigma}_{\boldsymbol{Q}}$ are defined in (3.3) and (3.4).

We defer the proof of Lemma 3.6 to [29]. Lemma 3.6 implies that when the gradient becomes small, the value of the objective function is close to $C\left(\boldsymbol{K}^{*}\right)$. Now we consider the smoothness condition of the objective function. Recall that a function $f: \mathbb{R}^{n} \rightarrow \mathbb{R}$ is said to be smooth if

$$
\left|f(x)-f(y)-\nabla f(y)^{\top}(x-y)\right| \leq \frac{M}{2}\|x-y\|^{2} \quad \forall x, y \in \mathbb{R}^{n},
$$

for some finite constant $M$. In general, it is difficult to characterize the smoothness of $C(\boldsymbol{K})$, since it may blow up when $A-B K_{t}$ is large. Here we will prove that $C(\boldsymbol{K})$ is "almost" smooth, in the sense that when $\boldsymbol{K}^{\prime}$ is sufficiently close to $\boldsymbol{K}, C\left(\boldsymbol{K}^{\prime}\right)-C(\boldsymbol{K})$ is bounded by the sum of the first and second order terms in $\boldsymbol{K}-\boldsymbol{K}^{\prime}$.

Lemma 3.7 ("almost smoothness"). Let $\left\{x_{t}^{\prime}\right\}$ be the sequence of states for a single trajectory generated by $\boldsymbol{K}^{\prime}$ starting from $x_{0}^{\prime}=x_{0}$. Then, $C(\boldsymbol{K})$ satisfies

$$
\begin{aligned}
C\left(\boldsymbol{K}^{\prime}\right)-C(\boldsymbol{K})=\sum_{t=0}^{T-1} & {\left[2 \operatorname{Tr}\left(\Sigma_{t}^{\prime}\left(K_{t}^{\prime}-K_{t}\right)^{\top} E_{t}\right)\right.} \\
& \left.+\operatorname{Tr}\left(\Sigma_{t}^{\prime}\left(K_{t}^{\prime}-K_{t}\right)^{\top}\left(R_{t}+B^{\top} P_{t+1} B\right)\left(K_{t}^{\prime}-K_{t}\right)\right)\right]
\end{aligned}
$$

where $\Sigma_{t}^{\prime}=\mathbb{E}\left[x_{t}^{\prime}\left(x_{t}^{\prime}\right)^{\top}\right]$.

We defer the proof of Lemma 3.7 to [29]. To see why Lemma 3.7 is related to the smoothness, observe that when $\boldsymbol{K}^{\prime}$ is sufficiently close to $\boldsymbol{K}$, in the sense that

$$
\Sigma_{t}^{\prime} \approx \Sigma_{t}+O\left(\left\|K_{t}-K_{t}^{\prime}\right\|\right) \quad \forall t=0,1, \ldots, T-1,
$$

the first term in (3.12) will behave as $\operatorname{Tr}\left(\left(K_{t}-K_{t}^{\prime}\right) \nabla_{t} C(\boldsymbol{K})\right)$ by Lemma 3.5, and the second term in (3.12) will be of second order in $K_{t}-K_{t}^{\prime}$.

To utilize Lemmas 3.6 and 3.7 in the proof of Theorem 3.3, we need to further bound $P_{t}$ and $\Sigma_{\boldsymbol{K}}$, which is provided below in Lemma 3.8. The proof can be found in [29].

Lemma 3.8. Assume that Assumption 2.1 holds, and $\underline{\sigma}_{\boldsymbol{X}}>0$. Then we have

$$
\left\|P_{t}\right\| \leq \frac{C(\boldsymbol{K})}{\underline{\sigma}_{\boldsymbol{X}}}, \quad\left\|\Sigma_{\boldsymbol{K}}\right\| \leq \frac{C(\boldsymbol{K})}{\underline{\sigma}_{Q}}
$$

where $\underline{\sigma}_{\boldsymbol{X}}$ and $\underline{\sigma}_{\boldsymbol{Q}}$ are defined as in (3.3) and (3.5).

Copyright $@$ by SIAM. Unauthorized reproduction of this article is prohibited. 
3.2. Perturbation analysis of $\Sigma_{K}$. First, let us define two linear operators on symmetric matrices. For $X \in \mathbb{R}^{d \times d}$ we set

$$
\begin{aligned}
\mathcal{F}_{K_{t}}(X) & =\left(A-B K_{t}\right) X\left(A-B K_{t}\right)^{\top}, \\
\mathcal{T}_{\boldsymbol{K}}(X) & :=X+\sum_{t=0}^{T-1} \Pi_{i=0}^{t}\left(A-B K_{i}\right) X \Pi_{i=0}^{t}\left(A-B K_{t-i}\right)^{\top} .
\end{aligned}
$$

If we write $\mathcal{G}_{t}=\mathcal{F}_{K_{t}} \circ \mathcal{F}_{K_{t-1}} \circ \cdots \circ \mathcal{F}_{K_{0}}$, then

$$
\begin{aligned}
\mathcal{G}_{t}(X) & =\mathcal{F}_{K_{t}} \circ \mathcal{G}_{t-1}(X)=\Pi_{i=0}^{t}\left(A-B K_{i}\right) X \Pi_{i=0}^{t}\left(A-B K_{t-i}\right)^{\top} \\
\mathcal{T}_{\boldsymbol{K}}(X) & =X+\sum_{t=0}^{T-1} \mathcal{G}_{t}(X) .
\end{aligned}
$$

We first show the relationship between the operator $\mathcal{T}_{\boldsymbol{K}}$ and the quantity $\Sigma_{\boldsymbol{K}}$. The proof can be found in [29].

Proposition 3.9. For $T \geq 2$, we have that

$$
\Sigma_{K}=\mathcal{T}_{\boldsymbol{K}}\left(\Sigma_{0}\right)+\Delta(\boldsymbol{K}, W)
$$

where $\Delta(\boldsymbol{K}, W)=\sum_{t=1}^{T-1} \sum_{s=1}^{t} D_{t, s} W D_{t, s}^{\top}+T W$, with $D_{t, s}=\Pi_{u=s}^{t}\left(A-B K_{u}\right)$ (for $s=1,2, \ldots, t)$, and $\Sigma_{0}=\mathbb{E}\left[x_{0} x_{0}^{\top}\right]$.

Let

$$
\rho:=\max \left\{\max _{0 \leq t \leq T-1}\left\|A-B K_{t}\right\|, \max _{0 \leq t \leq T-1}\left\|A-B K_{t}^{\prime}\right\|, 1+\xi\right\}
$$

for some small constant $\xi>0$. Then we have the following result on perturbations of $\Sigma_{K}$

Lemma 3.10 (perturbation analysis of $\Sigma_{K}$ ). Assume Assumption 2.1 holds. Then

$$
\begin{aligned}
\left\|\Sigma_{\boldsymbol{K}}-\Sigma_{\boldsymbol{K}^{\prime}}\right\| & \leq\left\|\left(\mathcal{T}_{\boldsymbol{K}}-\mathcal{T}_{\boldsymbol{K}^{\prime}}\right)\left(\Sigma_{0}\right)\right\|+\left\|\Delta(\boldsymbol{K}, W)-\Delta\left(\boldsymbol{K}^{\prime}, W\right)\right\| \\
& \leq \frac{\rho^{2 T}-1}{\rho^{2}-1}\left(\frac{C(\boldsymbol{K})}{\underline{\sigma}_{Q}}+T\|W\|\right)\left(2 \rho\|B\|\left\|\boldsymbol{K}-\boldsymbol{K}^{\prime}\right\|+\|B\|^{2}\left\|\boldsymbol{K}-\boldsymbol{K}^{\prime}\right\|^{2}\right) .
\end{aligned}
$$

Remark 3.11. By the definition of $\rho$ in (3.16), we have $\rho \geq 1+\xi>1$. This regularization term $1+\xi$ is defined for ease of exposition. Alternatively, if we define $\rho:=\max \left\{\max _{0 \leq t \leq T-1}\left\|A-B K_{t}\right\|, \max _{0 \leq t \leq T-1}\left\|A-B K_{t}^{\prime}\right\|\right\}$, a similar analysis can still be carried out by considering the different cases: $\rho<1, \rho=1$, and $\rho>1$. Note that for the infinite horizon problem, the spectral radius of $A-B K$ needs to be smaller than 1 to guarantee the stability of the system (see [22]). In our setting with finite horizon, instability is not an issue and we do not need a condition on the boundedness of $\rho$. However, we will show later that $\rho$ does appear in the sample complexity results. The smaller the $\rho$, the smaller the sample complexity.

The proof of Lemma 3.10 is based on Lemmas 3.12 and 3.13 below, which establish the Lipschitz property for the operators $\mathcal{F}_{K_{t}}$ and $\mathcal{G}_{t}$, respectively.

Lemma 3.12. It holds that $\forall t=0,1, \ldots, T-1$,

$$
\left\|\mathcal{F}_{K_{t}}-\mathcal{F}_{K_{t}^{\prime}}\right\| \leq 2\left\|A-B K_{t}\right\|\|B\|\left\|K_{t}-K_{t}^{\prime}\right\|+\|B\|^{2}\left\|K_{t}-K_{t}^{\prime}\right\|^{2} .
$$

Copyright (c) by SIAM. Unauthorized reproduction of this article is prohibited. 
We refer the reader to [22, Lemma 19] for the proof of Lemma 3.12.

Recall the definition of $\mathcal{G}_{t}$ in (3.13) associated with $\boldsymbol{K}$; similarly let us define $\mathcal{G}_{t}^{\prime}=\mathcal{F}_{K_{t}^{\prime}} \circ \mathcal{F}_{K_{t-1}^{\prime}} \circ \cdots \circ \mathcal{F}_{K_{0}^{\prime}}$ for policy $\boldsymbol{K}^{\prime}$. Then we have the following perturbation analysis for $\mathcal{G}_{t}$.

LEMma 3.13 (perturbation analysis for $\mathcal{G}_{t}$ ). For any symmetric matrix $\Sigma \in \mathbb{R}^{d \times d}$, we have that

$$
\sum_{t=0}^{T-1}\left\|\left(\mathcal{G}_{t}-\mathcal{G}_{t}^{\prime}\right)(\Sigma)\right\| \leq \frac{\rho^{2 T}-1}{\rho^{2}-1}\left(\sum_{t=0}^{T-1}\left\|\mathcal{F}_{K_{t}}-\mathcal{F}_{K_{t}^{\prime}}\right\|\right)\|\Sigma\| .
$$

We defer the proof of Lemma 3.13 to [29]. The following perturbation analysis on $\mathcal{T}$ follows immediately from Lemma 3.13.

COROLlary 3.14. For any symmetric matrix $\Sigma \in \mathbb{R}^{d \times d}$, we have

$$
\left\|\left(\mathcal{T}_{\boldsymbol{K}}-\mathcal{T}_{\boldsymbol{K}^{\prime}}\right)(\Sigma)\right\| \leq \frac{\rho^{2 T}-1}{\rho^{2}-1}\left(\sum_{t=0}^{T-1}\left\|\mathcal{F}_{K_{t}}-\mathcal{F}_{K_{t}^{\prime}}\right\|\right)\|\Sigma\|,
$$

where $\rho$ is defined as in (3.16).

Now we are ready for the proof of Lemma 3.10.

Proof of Lemma 3.10. Using Lemma 3.12,

$$
\begin{aligned}
\sum_{t=0}^{T-1}\left\|\mathcal{F}_{K_{t}}-\mathcal{F}_{K_{t}^{\prime}}\right\| & =\sum_{t=0}^{T-1}\left(2\left\|A-B K_{t}\right\|\|B\|\left\|K_{t}-K_{t}^{\prime}\right\|+\|B\|^{2}\left\|K_{t}-K_{t}^{\prime}\right\|^{2}\right) \\
& \leq 2 \rho\|B\| \sum_{t=0}^{T-1}\left\|K_{t}-K_{t}^{\prime}\right\|+\|B\|^{2} \sum_{t=0}^{T-1}\left\|K_{t}-K_{t}^{\prime}\right\|^{2} .
\end{aligned}
$$

In the same way as for the proof of Lemma 3.13, we have, $\forall t=1, \ldots, T-1$,

$$
\sum_{s=1}^{t}\left\|D_{t, s} W D_{t, s}^{\top}-D_{t, s}^{\prime} W\left(D_{t, s}^{\prime}\right)^{\top}\right\| \leq \frac{\rho^{2 T}-1}{\rho^{2}-1}\left(\sum_{s=0}^{t}\left\|\mathcal{F}_{K_{s}}-\mathcal{F}_{K_{s}^{\prime}}\right\|\right)\|W\| .
$$

By Proposition 3.9, Corollary 3.14, (3.14), and (3.20), we have

$$
\begin{aligned}
\left\|\Sigma_{\boldsymbol{K}}-\Sigma_{\boldsymbol{K}^{\prime}}\right\| & \leq\left\|\left(\mathcal{T}_{\boldsymbol{K}}-\mathcal{T}_{\boldsymbol{K}^{\prime}}\right)\left(\Sigma_{0}\right)\right\|+\sum_{t=1}^{T-1} \sum_{s=1}^{t}\left\|D_{t, s} W D_{t, s}^{\top}-D_{t, s}^{\prime} W\left(D_{t, s}^{\prime}\right)^{\top}\right\| \\
& \leq \frac{\rho^{2 T}-1}{\rho^{2}-1}\left(\sum_{t=0}^{T-1}\left\|\mathcal{F}_{K_{t}}-\mathcal{F}_{K_{t}^{\prime}}\right\|\right)\left(\left\|\Sigma_{0}\right\|+T\|W\|\right) \\
& \leq \frac{\rho^{2 T}-1}{\rho^{2}-1}\left(\frac{C(\boldsymbol{K})}{\underline{\sigma_{Q}}}+T\|W\|\right)\left(2 \rho\|B\|\left\|\boldsymbol{K}-\boldsymbol{K}^{\prime}\right\|+\|B\|^{2}\left\|\boldsymbol{K}-\boldsymbol{K}^{\prime}\right\|^{2}\right) .
\end{aligned}
$$

The last inequality holds since $\left\|\Sigma_{0}\right\| \leq\left\|\Sigma_{\boldsymbol{K}}\right\| \leq \frac{C(\boldsymbol{K})}{\underline{\sigma}_{Q}}$ by Lemma 3.8.

3.3. Convergence and complexity analysis. We now provide the proof of Theorem 3.3 after two preliminary lemmas.

Copyright (c) by SIAM. Unauthorized reproduction of this article is prohibited. 
Lemma 3.15. Assume Assumption 2.1 holds, $\underline{\sigma}_{\boldsymbol{X}}>0$, and

$$
K_{t}^{\prime}=K_{t}-\eta \nabla_{t} C(\boldsymbol{K})
$$

where

$$
\eta \leq \min \left\{\frac{\left(\rho^{2}-1\right) \underline{\sigma}_{\boldsymbol{Q}} \underline{\sigma}_{\boldsymbol{X}}}{2 T\left(\rho^{2 T}-1\right)(2 \rho+1)\left(C(\boldsymbol{K})+\underline{\sigma}_{\boldsymbol{Q}} T\|W\|\right)\|B\| \max _{t}\left\{\left\|\nabla_{t} C(\boldsymbol{K})\right\|\right\}}, \frac{1}{2 C_{1}}\right\},
$$

with

$$
\begin{aligned}
C_{1}= & \left(\frac{C(\boldsymbol{K})}{\underline{\sigma}_{\boldsymbol{Q}}}+T\|W\|\right)\left(\frac{(2 \rho+1)\|B\|\left(\rho^{2 T}-1\right)}{\left(\rho^{2}-1\right) \underline{\sigma}_{\boldsymbol{X}}} \sum_{t=0}^{T-1}\left\|\nabla_{t} C(\boldsymbol{K})\right\|\right) \\
& +\frac{2 C(\boldsymbol{K})}{\underline{\sigma}_{\boldsymbol{Q}}} \sum_{t=0}^{T-1}\left\|R_{t}+B^{\top} P_{t+1} B\right\| .
\end{aligned}
$$

Then we have

$$
C\left(\boldsymbol{K}^{\prime}\right)-C\left(\boldsymbol{K}^{*}\right) \leq\left(1-2 \eta \underline{\sigma}_{\boldsymbol{R}} \frac{\underline{\sigma}_{\boldsymbol{X}}^{2}}{\left\|\Sigma_{\boldsymbol{K}^{*}}\right\|}\right)\left(C(\boldsymbol{K})-C\left(\boldsymbol{K}^{*}\right)\right) .
$$

We defer the proof of Lemma 3.15 to [29].

Lemma 3.16. Assume Assumption 2.1 holds and $\underline{\sigma}_{\boldsymbol{X}}>0$. Then we have that

$$
\sum_{t=0}^{T-1}\left\|\nabla_{t} C(\boldsymbol{K})\right\|^{2} \leq 4\left(\frac{C(\boldsymbol{K})}{\underline{\sigma}_{\boldsymbol{Q}}}\right)^{2} \frac{\max _{t}\left\|R_{t}+B^{\top} P_{t+1} B\right\|}{\underline{\sigma}_{\boldsymbol{X}}}\left(C(\boldsymbol{K})-C\left(\boldsymbol{K}^{*}\right)\right),
$$

and that

$$
\sum_{t=0}^{T-1}\left\|K_{t}\right\| \leq \frac{1}{\underline{\sigma}_{\boldsymbol{R}}}\left(\sqrt{T \cdot \frac{\max _{t}\left\|R_{t}+B^{\top} P_{t+1} B\right\|}{\underline{\sigma}_{\boldsymbol{X}}}\left(C(\boldsymbol{K})-C\left(\boldsymbol{K}^{*}\right)\right)}+\sum_{t=0}^{T-1}\left\|B^{\top} P_{t+1} A\right\|\right) .
$$

Proof. Using Lemma 3.8 we have

$$
\begin{aligned}
\sum_{t=0}^{T-1}\left\|\nabla_{t} C(\boldsymbol{K})\right\|^{2} & \leq 4 \sum_{t=0}^{T-1} \operatorname{Tr}\left(\Sigma_{t} E_{t}^{\top} E_{t} \Sigma_{t}\right) \leq 4 \sum_{t=0}^{T-1}\left\|\Sigma_{t}\right\|^{2} \operatorname{Tr}\left(E_{t}^{\top} E_{t}\right) \\
& \leq 4\left(\frac{C(\boldsymbol{K})}{\underline{\sigma}_{Q}}\right)^{2} \sum_{t=0}^{T-1} \operatorname{Tr}\left(E_{t}^{\top} E_{t}\right) .
\end{aligned}
$$

From Lemma 3.6 we have

$$
\begin{aligned}
C(\boldsymbol{K})-C\left(\boldsymbol{K}^{*}\right) & \geq \underline{\sigma}_{\boldsymbol{X}} \sum_{t=0}^{T-1} \frac{1}{\left\|R_{t}+B^{\top} P_{t+1} B\right\|} \operatorname{Tr}\left(E_{t}^{\top} E_{t}\right) \\
& \geq \frac{\underline{\sigma}_{\boldsymbol{X}}}{\max _{t}\left\|R_{t}+B^{\top} P_{t+1} B\right\|} \sum_{t=0}^{T-1} \operatorname{Tr}\left(E_{t}^{\top} E_{t}\right),
\end{aligned}
$$

Copyright (c) by SIAM. Unauthorized reproduction of this article is prohibited. 
and hence

$$
\sum_{t=0}^{T-1}\left\|\nabla_{t} C(\boldsymbol{K})\right\|^{2} \leq 4\left(\frac{C(\boldsymbol{K})}{\underline{\sigma}_{Q}}\right)^{2} \frac{\max _{t}\left\|R_{t}+B^{\top} P_{t+1} B\right\|}{\underline{\sigma}_{\boldsymbol{X}}}\left(C(\boldsymbol{K})-C\left(\boldsymbol{K}^{*}\right)\right) .
$$

For the second claim, using Lemma 3.6 again,

$$
\begin{aligned}
\sum_{t=0}^{T-1}\left\|K_{t}\right\| & =\sum_{t=0}^{T-1}\left\|\left(R_{t}+B^{\top} P_{t+1} B\right)^{-1} K_{t}\left(R_{t}+B^{\top} P_{t+1} B\right)\right\| \\
& \leq \sum_{t=0}^{T-1} \frac{1}{\sigma_{\min }\left(R_{t}\right)}\left\|K_{t}\left(R_{t}+B^{\top} P_{t+1} B\right)\right\| \leq \sum_{t=0}^{T-1} \frac{1}{\sigma_{\min }\left(R_{t}\right)}\left(\left\|E_{t}\right\|+\left\|B^{\top} P_{t+1} A\right\|\right) \\
& \leq \sum_{t=0}^{T-1}\left(\frac{\sqrt{\operatorname{Tr}\left(E_{t}^{\top} E_{t}\right)}}{\sigma_{\min }\left(R_{t}\right)}+\frac{\left\|B^{\top} P_{t+1} A\right\|}{\sigma_{\min }\left(R_{t}\right)}\right) \\
& \leq \frac{1}{\underline{\sigma}_{\boldsymbol{R}}}\left(\sqrt{T \cdot \sum_{t=0}^{T-1} \operatorname{Tr}\left(E_{t}^{\top} E_{t}\right)}+\sum_{t=0}^{T-1}\left\|B^{\top} P_{t+1} A\right\|\right) \\
& \leq \frac{1}{\underline{\sigma}_{\boldsymbol{R}}}\left(\sqrt{T \cdot \frac{\max _{t}\left\|R_{t}+B^{\top} P_{t+1} B\right\|}{\underline{\sigma}_{\boldsymbol{X}}}\left(C(\boldsymbol{K})-C\left(\boldsymbol{K}^{*}\right)\right)}+\sum_{t=0}^{T-1}\left\|B^{\top} P_{t+1} A\right\|\right)
\end{aligned}
$$

The second inequality holds by the definition of $E_{t}$ in (3.11), the next to last step uses the Cauchy-Schwarz inequality, and the last inequality holds by (3.25).

Proof of Theorem 3.3. In order to show the existence of a positive $\eta$ such that (3.23) holds, it suffices to show there exists a positive lower bound on the right-hand side of (3.23). By Lemma 3.16 and the Cauchy-Schwarz inequality,

$$
\begin{aligned}
\sum_{t=0}^{T-1}\left\|\nabla_{t} C(\boldsymbol{K})\right\| & \leq \sqrt{T \cdot \sum_{t=0}^{T-1}\left\|\nabla_{t} C(\boldsymbol{K})\right\|^{2}} \\
& \leq \sqrt{4 T \cdot\left(\frac{C(\boldsymbol{K})}{\underline{\sigma}_{\boldsymbol{Q}}}\right)^{2} \frac{\max _{t}\left\|R_{t}+B^{\top} P_{t+1} B\right\|}{\underline{\sigma}_{\boldsymbol{X}}}\left(C(\boldsymbol{K})-C\left(\boldsymbol{K}^{*}\right)\right)}
\end{aligned}
$$

Note that if $d<a b+c$ for some $a>0, b>0, c>0$, and $d>0$, then $\frac{1}{d}>$ $\frac{1}{(a+1)(b+1)(c+1)}$. Also $\frac{1}{a^{n}+1}>\frac{1}{(a+1)^{n}}$ for $a>0$ and $n \in \mathbb{N}^{+}$. Therefore, based on (3.24) and (3.26), $\frac{1}{C_{1}}$ is bounded below by polynomials in $\frac{1}{\rho}, \frac{1}{C(\boldsymbol{K})+1}, \frac{1}{\|B\|+1}, \frac{1}{\|\boldsymbol{R}\|+1}$, $\frac{1}{\|W\|+1}, \underline{\sigma}_{\boldsymbol{X}}, \underline{\sigma}_{\boldsymbol{Q}}, \frac{1}{\underline{\sigma}_{\boldsymbol{X}}+1}$, and $\frac{1}{\underline{\sigma}_{\boldsymbol{Q}}+1}$.

Now we aim to show that $\frac{1}{\rho}$ is bounded below by some polynomials in the parameters. To see this, let us first show that $\rho$ is bounded above by polynomials in $\|A\|$, $\|B\|,\|\boldsymbol{R}\|, \frac{1}{\underline{\sigma}_{\boldsymbol{X}}}, \frac{1}{\underline{\sigma}_{\boldsymbol{R}}}$, and $C(\boldsymbol{K})$. Since $\|B\|\left\|K_{t}^{\prime}-K_{t}\right\| \leq \frac{\underline{\sigma}_{\boldsymbol{Q}} \underline{\underline{\sigma}}_{\boldsymbol{X}}}{4 C(\boldsymbol{K})} \leq \frac{1}{2}$ holds under the assumptions in Lemma 3.15, we have

$$
\max _{0 \leq t \leq T-1}\left\|A-B K_{t}^{\prime}\right\| \leq \max _{0 \leq t \leq T-1}\left(\left\|A-B K_{t}\right\|+\|B\|\left\|K_{t}^{\prime}-K_{t}\right\|\right) \leq \max _{0 \leq t \leq T-1}\left\|A-B K_{t}\right\|+\frac{1}{2}
$$

Copyright (c) by SIAM. Unauthorized reproduction of this article is prohibited. 
thus

$$
\begin{aligned}
\rho & =\max \left\{\max _{0 \leq t \leq T-1}\left\|A-B K_{t}\right\|, \max _{0 \leq t \leq T-1}\left\|A-B K_{t}^{\prime}\right\|, 1+\xi\right\} \\
& \leq \max \left\{\max _{0 \leq t \leq T-1}\left\|A-B K_{t}\right\|+\frac{1}{2}, 1+\xi\right\} \leq \max \left\{\|A\|+\|B\| \sum_{t=0}^{T-1}\left\|K_{t}\right\|+\frac{1}{2}, 1+\xi\right\} .
\end{aligned}
$$

Given the bound on $\sum_{t=0}^{T-1}\left\|K_{t}\right\|$ by Lemma 3.16 and $\left\|P_{t}\right\| \leq \frac{C(\boldsymbol{K})}{\underline{\sigma}_{\boldsymbol{X}}}$ by Lemma $3.8, \rho$ is bounded above by polynomials in $\|A\|,\|B\|,\|R\|, \frac{1}{\underline{\sigma}_{X}}, \frac{1}{\sigma_{\boldsymbol{R}}}$, and $C(\boldsymbol{K})$, or a constant $1+\xi$. Therefore $\frac{1}{\rho}$ is bounded below by polynomials in $\frac{1}{\|A\|+1}, \frac{1}{\|B\|+1}, \frac{1}{\|\boldsymbol{R}\|+1}, \underline{\sigma}_{\boldsymbol{X}}$, $\underline{\sigma}_{\boldsymbol{R}}$, and $\frac{1}{C(\boldsymbol{K})+1}$, or a constant $\frac{1}{1+\xi}$. Hence, by choosing $\eta \in \mathcal{H}\left(\frac{1}{C\left(\boldsymbol{K}^{0}\right)+1}\right)$ to be an appropriate polynomial in $\frac{1}{C\left(\boldsymbol{K}^{0}\right)}, \frac{1}{C\left(\boldsymbol{K}^{0}\right)+1}, \frac{1}{\|A\|+1}, \frac{1}{\|B\|+1}, \frac{1}{\|\boldsymbol{R}\|+1}, \frac{1}{\|W\|+1}, \underline{\sigma}_{\boldsymbol{X}}$, $\underline{\sigma}_{\boldsymbol{Q}}, \underline{\sigma}_{\boldsymbol{R}}, \frac{1}{\underline{\sigma}_{\boldsymbol{X}}+1}$, and $\frac{1}{\underline{\sigma}_{\boldsymbol{Q}}+1},(3.23)$ is satisfied, since by performing gradient descent, $C\left(\boldsymbol{K}^{1}\right)<C\left(\boldsymbol{K}^{0}\right)$. Therefore, by Lemma 3.15 , we have

$$
C\left(\boldsymbol{K}^{1}\right)-C\left(\boldsymbol{K}^{*}\right) \leq\left(1-2 \eta \underline{\sigma}_{\boldsymbol{R}} \frac{\underline{\sigma}_{\boldsymbol{X}}^{2}}{\left\|\Sigma_{\boldsymbol{K}^{*}}\right\|}\right)\left(C\left(\boldsymbol{K}^{0}\right)-C\left(\boldsymbol{K}^{*}\right)\right),
$$

which implies that the cost decreases at $n=1$. Suppose that $C\left(\boldsymbol{K}^{n}\right) \leq C\left(\boldsymbol{K}^{0}\right)$; then the step size condition in (3.23) is still satisfied by Lemma 3.16. Thus, Lemma 3.15 can again be applied for the update at round $n+1$ to obtain

$$
C\left(\boldsymbol{K}^{n+1}\right)-C\left(\boldsymbol{K}^{*}\right) \leq\left(1-2 \eta \underline{\sigma}_{\boldsymbol{R}} \frac{\underline{\sigma}_{\boldsymbol{X}}^{2}}{\left\|\Sigma_{\boldsymbol{K}^{*}}\right\|}\right)\left(C\left(\boldsymbol{K}^{n}\right)-C\left(\boldsymbol{K}^{*}\right)\right) .
$$

For $\epsilon>0$, provided $N \geq \frac{\left\|\Sigma_{\boldsymbol{K}^{*}}\right\|}{2 \eta \underline{\boldsymbol{X}}^{2} \underline{\sigma}_{\boldsymbol{R}}} \log \frac{C\left(\boldsymbol{K}^{0}\right)-C\left(\boldsymbol{K}^{*}\right)}{\epsilon}$, we have

$$
C\left(\boldsymbol{K}^{N}\right)-C\left(\boldsymbol{K}^{*}\right) \leq \epsilon .
$$

4. Sample-based policy gradient method with unknown parameters. In the setting with unknown parameters, the controller has only simulation access to the model; the model parameters, $A, B,\left\{Q_{t}\right\}_{t=0}^{T},\left\{R_{t}\right\}_{t=0}^{T-1}$, are unknown. By using a zeroth-order optimization method to approximate the gradient, this section proves the policy gradient method with unknown parameters also leads to a global optimal policy, with both polynomial computational and sample complexities.

Note that in this section, when bounding the Frobenius norm of a matrix, we usually treat the matrix as a stacked vector. Therefore we denote by $D=k \times d$ the dimension of the corresponding vector formed from the $\boldsymbol{K}$ matrix for convenience in the proofs. Therefore in each iteration $n=1,2, \ldots, N$, we can update the policy as, for $t=0,1, \ldots, T-1$,

$$
K_{t}^{n+1}=K_{t}^{n}-\eta \nabla_{t} \widehat{C\left(\boldsymbol{K}^{n}\right)},
$$

where $\left.\nabla_{t} \widehat{C\left(\boldsymbol{K}^{n}\right.}\right)$ is the estimate of $\nabla_{t} C\left(\boldsymbol{K}^{n}\right)$. We analyze Algorithm 4.1 below.

Remark 4.1 (zeroth-order optimization approach in the subroutine (4.2)). In the estimation of the gradient term (4.2), we adopt a zeroth-order optimization method, using only query access to a sample of the reward function $c(\cdot)$ at input points $K$, 


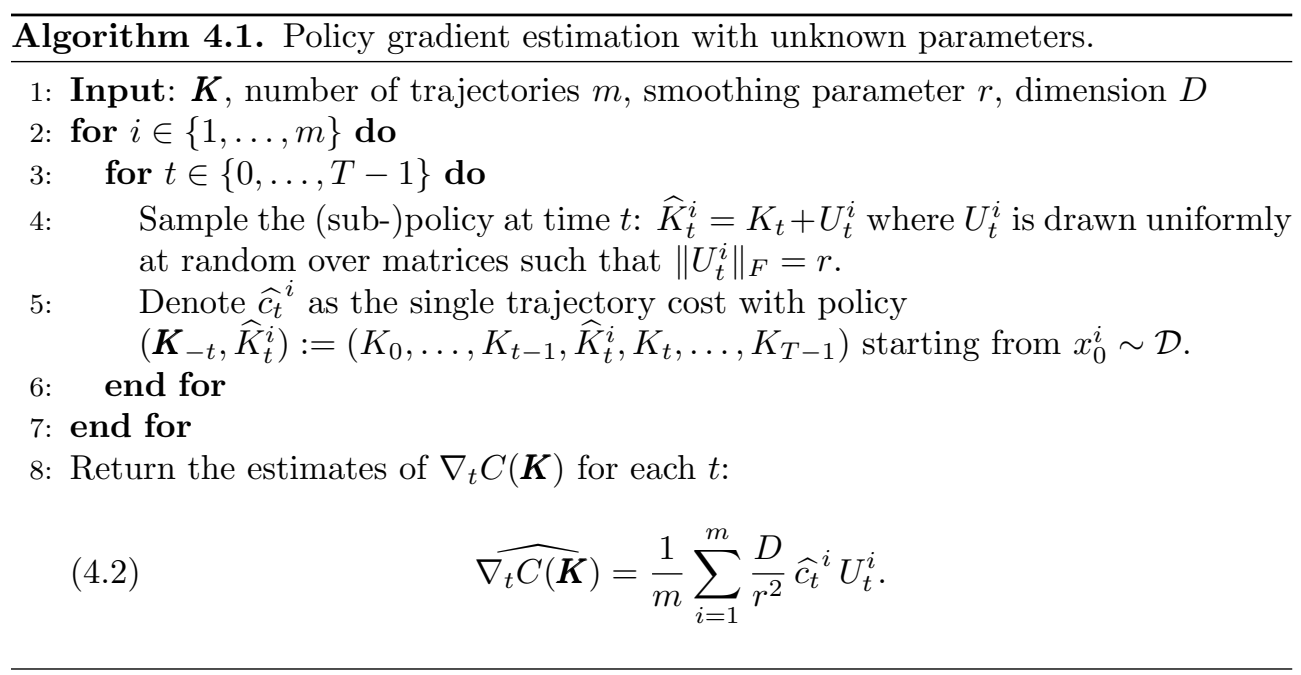

without querying the gradients and higher order derivatives of $c(\cdot)$. In a way similar to the observation in [22], the objective $C(\boldsymbol{K})$ may not be finite for every policy $\boldsymbol{K}$ when Gaussian smoothing is applied; therefore $\mathbb{E}_{\boldsymbol{U} \sim \mathcal{N}\left(0, \sigma^{2} I\right)}[C(\boldsymbol{K}+\boldsymbol{U})]$ may not be well-defined. This is avoidable by smoothing over the surface of a ball. The step (4.2) (in Algorithm 4.1) provides a procedure to find a (bounded bias) estimate $\nabla \widehat{C(\boldsymbol{K})}$ of $\nabla C(\boldsymbol{K})$.

The idea in (4.2) is to approximate the gradient of a function by only using the function values (see, e.g., Lemma 2.1 in [24]). Observe that by a Taylor expansion to first order, $\mathbb{E}[f(x+U) U] \approx \mathbb{E}[(\nabla f(x) \cdot U) U]=\nabla f(x) r^{2} / D$ when $x \in \mathbb{R}^{D}$ and $U$ is uniform over the surface of the ball of radius $r$ in $\mathbb{R}^{D}$. Thus the gradient of the function $f$ at $x$ can be estimated by averaging over the samples $\frac{D}{r^{2}} f(x+U) U$.

Note that in Algorithm 4.1, we require $m N T^{2}$ samples to perform the policy gradient method $N$ times.

To guarantee the global convergence of the sample-based algorithm (Algorithm 4.1 ), we propose some conditions on the distribution of $x_{0}$ and $\left\{w_{t}\right\}_{t=0}^{T-1}$, in addition to the finite second moment condition specified in section 2 .

Definition 4.2. A zero-mean random variable $X$

1. is said to be sub-Gaussian with variance proxy $\sigma^{2}$, and we write $X \in S G\left(\sigma^{2}\right)$ if its moment generating function satisfies $\mathbb{E}[\exp (\lambda X)] \leq \exp \left(\frac{\lambda^{2} \sigma^{2}}{2}\right)$ for all $\lambda \in \mathbb{R}$

2. is said to be subexponential with parameters $\left(\nu^{2}, \alpha\right)$, and we write $X \in S E\left(\nu^{2}, \alpha\right)$ if $\mathbb{E}[\exp (\lambda X)] \leq \exp \left(\frac{\lambda^{2} \nu^{2}}{2}\right)$ for any $\lambda$ such that $|\lambda| \leq \frac{1}{\alpha}$.

We assume the initial distribution and the noise in the state process dynamics satisfy the following assumptions.

Assumption 4.3 (initial state and noise process (II)).

1. Initial state: $x_{0}=\widetilde{W}_{0} z_{0}$, where $z_{0}=\left(z_{0,1}, \ldots, z_{0, d}\right) \in \mathbb{R}^{d}$ is a random vector with independent components $z_{0, i}$ which are sub-Gaussian, mean-zero, and have sub-Gaussian parameter $\sigma_{0}^{2} ; \widetilde{W}_{0} \in \mathbb{R}^{d \times d}$ is an unknown and deterministic matrix.

2. Noise process: $w_{t}=\widetilde{W} v_{t}$, where $v_{t}:=\left(v_{t, 1}, \ldots, v_{t, d}\right) \in \mathbb{R}^{d}$ are i.i.d. and inde- 
pendent of $x_{0} . v_{t}$ has independent components $v_{t, i}$ which are sub-Gaussian, mean-zero, and have sub-Gaussian parameter $\sigma_{w}^{2} \forall t=0,1, \ldots, T-1 . \widetilde{W} \in$ $\mathbb{R}^{d \times d}$ is an unknown and deterministic matrix.

Note that Assumptions 3.1 and 4.3 serve different purposes in this paper. Assumption 3.1 provides one sufficient condition to assure $\underline{\sigma}_{\boldsymbol{X}}>0$. Assumption 4.3 is used to guarantee the convergence of the sample-based algorithm (Algorithm 4.1).

In addition to the model parameters specified in section 3, here we assume $\mathcal{H}(\cdot)$ includes polynomials that are also functions of $\sigma_{0}, \frac{1}{\sigma_{0}}, \frac{1}{\sigma_{0}+1}, \sigma_{w}, \frac{1}{\sigma_{w}}, \frac{1}{\sigma_{w}+1}\|\widetilde{W}\|, \frac{1}{\|\widetilde{W}\|}$, $\frac{1}{\|\widetilde{W}\|+1},\left\|\widetilde{W}_{0}\right\|, \frac{1}{\left\|\widetilde{W}_{0}\right\|}$, and $\frac{1}{\left\|\widetilde{W}_{0}\right\|+1}$.

TheOrem 4.4. Assume Assumptions 2.1 and 4.3 hold, and further assume $\underline{\sigma}_{\boldsymbol{X}}>$ 0 and $C\left(\boldsymbol{K}^{0}\right)$ is finite. At every step the policy is updated as in (4.1), that is,

$$
K_{t}^{n+1}=K_{t}^{n}-\eta \nabla_{t}{\widehat{C\left(\boldsymbol{K}^{n}\right.}},
$$

with $\eta \in \mathcal{H}\left(\frac{1}{C\left(\boldsymbol{K}^{0}\right)+1}\right)$, and $\left.\nabla_{t} \widehat{C\left(\boldsymbol{K}^{n}\right.}\right)$ is computed with hyperparameters $(r, m)$ such that $r<1 / \bar{h}_{\text {radius }}$ and $m>\bar{h}_{\text {sample }}$ with some fixed polynomials $\bar{h}_{\text {radius }} \in \mathcal{H}\left(1 / \epsilon, \mathrm{C}\left(\boldsymbol{K}^{0}\right)\right)$ and $\bar{h}_{\text {sample }} \in \mathcal{H}\left(1 / \epsilon, \mathrm{C}\left(\boldsymbol{K}^{0}\right)\right)$. Then for $\epsilon>0$, if we have

$$
N \geq \frac{\left\|\Sigma_{\boldsymbol{K}^{*}}\right\|}{\eta \underline{\sigma}_{\boldsymbol{X}}^{2} \underline{\sigma}_{\boldsymbol{R}}} \log \frac{C\left(\boldsymbol{K}^{0}\right)-C\left(\boldsymbol{K}^{*}\right)}{\epsilon},
$$

it holds that $C\left(\boldsymbol{K}^{N}\right)-C\left(\boldsymbol{K}^{*}\right) \leq \epsilon$ with high probability (at least $1-\exp (-D)$ ).

Note that $\bar{h}_{\text {sample }}$ is quadratic in $1 / \epsilon$ (when the logarithmic order is omitted) and cubic in dimension $D$. The proof of Theorem 4.4 is based on a perturbation analysis of $C(\boldsymbol{K})$ and $\nabla_{t} C(\boldsymbol{K})$, smoothing, and the gradient descent analysis of the procedures in Algorithm 4.1. We provide the perturbation analysis and the smoothing analysis in sections 4.1 and 4.2, respectively. We defer the proof of Theorem 4.4 to section 4.3.

Projected policy gradient method. In many situations constrained optimization problems arise, and the projected gradient descent method is one popular approach to solve such problems. Recall that the projection of a point $\boldsymbol{y}=\left(y_{0}, \ldots, y_{T-1}\right)$ with $y_{t} \in \mathbb{R}^{k \times d}(t=0,1, \ldots, T-1)$ onto a set $\mathcal{S} \subset \mathbb{R}^{k \times(T \times d)}$ is defined as

$$
\Pi_{\mathcal{S}}(\boldsymbol{y})=\underset{\boldsymbol{x} \in \mathcal{S}}{\arg \min } \frac{1}{2} \sum_{t=0}^{T-1}\left\|x_{t}-y_{t}\right\|_{F}^{2} .
$$

Then the projected policy gradient (PPG) updating rule can be defined as

$$
\boldsymbol{K}^{n+1}=\Pi_{\mathcal{S}}\left(\boldsymbol{K}^{n}-\eta \nabla \widehat{C\left(\boldsymbol{K}^{n}\right)}\right),
$$

where $\left.\left.\widehat{\nabla C\left(\boldsymbol{K}^{n}\right.}\right)=\left(\widehat{\nabla_{0} C\left(\boldsymbol{K}^{n}\right.}\right), \ldots, \nabla_{T-1} C\left(\boldsymbol{K}^{n}\right)\right)$ denotes the estimate of $\nabla C\left(\boldsymbol{K}^{n}\right)$.

If the projection set $\mathcal{S}$ is convex and closed, the projection onto $\mathcal{S}$ is nonexpansive, that is, $\sum_{t=0}^{T-1}\left\|\widetilde{z}_{t}^{1}-\widetilde{z}_{t}^{2}\right\|_{F} \leq \sum_{t=0}^{T-1}\left\|z_{t}^{1}-z_{t}^{2}\right\|_{F}$ with $\widetilde{\boldsymbol{z}}^{1}=\Pi_{\mathcal{S}}\left(\boldsymbol{z}^{1}\right)$ and $\widetilde{\boldsymbol{z}}^{2}=\Pi_{\mathcal{S}}\left(\boldsymbol{z}^{2}\right)$. Given any policy matrix $K$ and learning rate $\eta$, define the gradient mapping for the projection operator,

$$
G(\boldsymbol{K}):=\frac{\Pi_{\mathcal{S}}(\boldsymbol{K}-\eta \nabla C(\boldsymbol{K}))-\boldsymbol{K}}{2 \eta},
$$


with $G(\boldsymbol{K})=\left(G_{0}(\boldsymbol{K}), \ldots, G_{T-1}(\boldsymbol{K})\right)$. Note that the gradient mapping has been commonly adopted in the analysis of projected gradient descent methods in constrained optimization $[36,47]$. A policy matrix $\widetilde{\boldsymbol{K}} \in \mathcal{S}$ is called a stationary point of $C(\cdot)$ if

$$
\nabla C(\widetilde{\boldsymbol{K}})^{\top}(\boldsymbol{K}-\widetilde{\boldsymbol{K}}) \leq 0 \quad \forall \boldsymbol{K} \in \mathcal{S} .
$$

It is well known in the optimization literature that (4.6) holds if and only if $G(\widetilde{\boldsymbol{K}})=0$. We have the following sublinear convergence result for the PPG version.

Theorem 4.5. Assume Assumptions 2.1 and 4.3 hold, and the projection set of policies, denoted by $\mathcal{S}$, is convex and closed. Further assume that $\boldsymbol{K}^{*} \in \mathcal{S}, \boldsymbol{K}^{0} \in \mathcal{S}$, $\underline{\sigma}_{\boldsymbol{X}}>0$, and $C\left(\boldsymbol{K}^{0}\right)$ is finite. At every step the policy is updated as in (4.4), that is,

$$
\left.\boldsymbol{K}^{n+1}=\Pi_{\mathcal{S}}\left(\boldsymbol{K}^{n}-\eta \nabla \widehat{\nabla\left(\boldsymbol{K}^{n}\right.}\right)\right)
$$

with $\eta \in \mathcal{H}\left(\frac{1}{C\left(\boldsymbol{K}^{0}\right)+1}\right)$, and $\left.\nabla_{t} \widehat{C\left(\boldsymbol{K}^{n}\right.}\right)(t=0,1, \ldots, T-1)$ is computed with hyperparameters $(r, m)$ such that $r<1 / \widehat{h}_{\text {radius }}$ and $m>\widehat{h}_{\text {sample }}$ with some fixed polynomials $\widehat{h}_{\text {radius }} \in \mathcal{H}\left(1 / \epsilon, \mathrm{C}\left(\boldsymbol{K}^{0}\right)\right)$ and $\widehat{h}_{\text {sample }} \in \mathcal{H}\left(1 / \epsilon, \mathrm{C}\left(\boldsymbol{K}^{0}\right)\right)$. Then the projected policy gradient method has a global sublinear convergence rate, that is,

$$
\left\{\frac{1}{N} \sum_{n=0}^{N-1}\left(\sum_{t=0}^{T-1}\left\|G_{t}\left(\boldsymbol{K}^{n}\right)\right\|_{F}^{2}\right)\right\}_{N \geq 1}
$$

converges to 0 at rate $\mathcal{O}\left(\frac{1}{N}\right)$, where $G_{t}(\boldsymbol{K})$ is defined in (4.5).

The proof of Theorem 4.5 can be found in [29].

Remark 4.6. We assume that the projection step is performed accurately, and that the associated computational cost is of separate interest and hence omitted here. The convergence result in Theorem 4.5 is described in terms of the sample complexity, and performing the projection step does not need extra samples.

4.1. Perturbation analysis of $C(K)$ and $\nabla_{t} C(K)$. This section shows that the objective function $C(\boldsymbol{K})$ and its gradient are stable with respect to small perturbations. The proofs of the following lemmas can be found in [29].

Lemma $4.7\left(C(\boldsymbol{K})\right.$ perturbation). Assume Assumptions 2.1 and 4.3 hold, $\underline{\sigma}_{\boldsymbol{X}}>$ 0 , and $\boldsymbol{K}^{\prime}$ is such that, $\forall t=0,1, \ldots, T-1$,

$$
\left\|K_{t}^{\prime}-K_{t}\right\| \leq \min \left\{\frac{\left(\rho^{2}-1\right) \underline{\sigma}_{\boldsymbol{Q}} \underline{\sigma}_{\boldsymbol{X}}}{2 T\left(\rho^{2 T}-1\right)(2 \rho+1)\left(C(\boldsymbol{K})+\underline{\sigma}_{\boldsymbol{Q}} T\|W\|\right)\|B\|},\left\|K_{t}\right\|, \frac{1}{T}\right\}
$$

where $\rho$ is defined as in (3.16). Then there exists a polynomial $h_{\text {cost }} \in \mathcal{H}(\mathrm{C}(\boldsymbol{K}))$ such that

$$
\left|C\left(\boldsymbol{K}^{\prime}\right)-C(\boldsymbol{K})\right| \leq h_{\text {cost }}\left\|\boldsymbol{K}^{\prime}-\boldsymbol{K}\right\| .
$$

Lemma $4.8\left(\nabla_{t} C(\boldsymbol{K})\right.$ perturbation). Under the same assumptions as in Lemma 4.7, there exists a polynomial $h_{\text {grad }} \in \mathcal{H}(\mathrm{C}(\boldsymbol{K}))$ such that

$$
\begin{aligned}
\left\|\nabla_{t} C\left(\boldsymbol{K}^{\prime}\right)-\nabla_{t} C(\boldsymbol{K})\right\| & \leq h_{\text {grad }}\left\|\boldsymbol{K}^{\prime}-\boldsymbol{K}\right\|, \\
\left\|\nabla_{t} C\left(\boldsymbol{K}^{\prime}\right)-\nabla_{t} C(\boldsymbol{K})\right\|_{F} & \leq h_{\text {grad }}\left\|\boldsymbol{K}^{\prime}-\boldsymbol{K}\right\|_{F} .
\end{aligned}
$$

Copyright $@$ by SIAM. Unauthorized reproduction of this article is prohibited. 
4.2. Smoothing and the gradient descent analysis. In this section, Lemma 4.9 provides the formula for the perturbed gradient term, Lemma 4.10 provides the concentration inequality for finite samples, and Lemma 4.11 provides the guarantees for the gradient approximation.

Recall that $D=k \times d$. Let $\mathbb{S}_{r}$ represent the uniform distribution over the points with norm $r$ in dimension $D$, and let $\mathbb{B}_{r}$ represent the uniform distribution over all points with norm at most $r$ in dimension $D$. For each $K_{t}(t=0,1, \ldots, T-1)$, the algorithm performs gradient descent on the following function:

$$
C_{t}^{r}(\boldsymbol{K})=\mathbb{E}_{V_{t} \sim \mathbb{B}_{r}}\left[C\left(\boldsymbol{K}+\boldsymbol{V}_{t}\right)\right],
$$

where $\boldsymbol{V}_{t}:=\left(0, \ldots, V_{t}, \ldots, 0\right)$ and $V_{t} \in \mathbb{R}^{k \times d}$.

Lemma 4.9. Assume $C(\boldsymbol{K})$ is finite; then

$$
\nabla_{t} C_{t}^{r}(\boldsymbol{K})=\frac{D}{r^{2}} \mathbb{E}_{U_{t} \sim \mathbb{S}_{r}}\left[C\left(\boldsymbol{K}+\boldsymbol{U}_{t}\right) U_{t}\right] .
$$

The proof of Lemma 4.9 is similar to the proof of [22, Lemma 29] and hence is omitted.

We first state two facts on sub-Gaussian and subexponential random variables. First, if $X$ and $Y$ are zero-mean independent random variables such that $X \in S G\left(\sigma_{x}^{2}\right)$ and $Y \in S G\left(\sigma_{y}^{2}\right)$, then $X Y \in S E\left(\sigma_{x} \sigma_{y}, 4 \sigma_{x} \sigma_{y}\right)$. Second, if $X_{1}, \ldots, X_{n}$ are zero-mean independent random variables such that $X_{i} \in S E\left(\nu_{i}^{2}, \alpha_{i}\right)$, then

$$
\sum_{i=1}^{n} X_{i} \in S E\left(\sum_{i=1}^{n} \nu_{i}^{2}, \max _{i} \alpha_{i}\right) .
$$

Using the above two facts, we have the following.

Lemma 4.10. Assume Assumptions 2.1 and 4.3 hold and $\underline{\sigma}_{\boldsymbol{X}}>0$; then there exist polynomials $\nu \in \mathcal{H}(\mathrm{C}(\boldsymbol{K}))$ and $\alpha \in \mathcal{H}(\mathrm{C}(\boldsymbol{K}))$ such that

$$
\left[\sum_{t=0}^{T-1}\left(x_{t}^{\top} Q_{t} x_{t}+u_{t}^{\top} R_{t} u_{t}\right)+x_{T}^{\top} Q_{T} x_{T}\right]
$$

is subexponential with parameter $\left(\nu^{2}, \alpha\right)$. Here $\left\{x_{t}\right\}_{t=0}^{T}$ is the dynamics under policy $K$.

Proof. We first observe that, by direct calculation,

$$
\left[\sum_{t=0}^{T-1}\left(x_{t}^{\top} Q_{t} x_{t}+u_{t}^{\top} R_{t} u_{t}\right)+x_{T}^{\top} Q_{T} x_{T}\right]=x_{0}^{\top} P_{0} x_{0}+\sum_{t=0}^{T-1} w_{t}^{\top} P_{t+1} w_{t} .
$$

Note that by (3.9) and Proposition 3.4, $P_{t}$ is symmetric and positive definite. The Frobenius norm $\|\cdot\|_{F}$ and the spectral norm $\|\cdot\|$ of the matrix $P_{t} \in \mathbb{R}^{d \times d}$ have the following property:

$$
\left\|P_{t}\right\| \leq\left\|P_{t}\right\|_{F} \leq \sqrt{d}\left\|P_{t}\right\| \quad \forall t=0,1, \ldots, T .
$$

Let $\widehat{\sigma}=\max \left\{\sigma_{0}, \sigma_{w}\right\}$. Given the Hanson-Wright inequality (Theorem 2.5 in [4]),

$$
\begin{aligned}
\mathbb{P}\left(\left|w_{t}^{\top} P_{t+1} w_{t}-\mathbb{E}\left[w_{t}^{\top} P_{t+1} w_{t}\right]\right| \geq t\right) \\
\quad=\mathbb{P}\left(\left|v_{t}^{\top}\left(\widetilde{W}^{\top} P_{t+1} \widetilde{W}\right) v_{t}-\mathbb{E}\left[v_{t}^{\top}\left(\widetilde{W}^{\top} P_{t+1} \widetilde{W}\right) v_{t}\right]\right| \geq t\right) \\
\leq 2 \exp \left(-c \min \left\{\frac{t^{2}}{2 \widehat{\sigma}^{4}\left\|\widetilde{W}^{\top} P_{t+1} \widetilde{W}\right\|_{F}^{2}}, \frac{t}{\widehat{\sigma}^{2}\left\|\widetilde{W}^{\top} P_{t+1} \widetilde{W}\right\|}\right\}\right)
\end{aligned}
$$

Copyright (c) by SIAM. Unauthorized reproduction of this article is prohibited. 
for some universal constant $c>0$ which is independent of $P_{t+1}$ and $w_{t}$.

Combining (4.11), (4.12), and Lemma 3.8,

$$
\begin{aligned}
\mathbb{P}\left(\mid w_{t}^{\top}\right. & \left.P_{t+1} w_{t}-\mathbb{E}\left[w_{t}^{\top} P_{t+1} w_{t}\right] \mid \geq t\right) \\
& \leq 2 \exp \left(-c \min \left\{\frac{t^{2}}{2 \widehat{\sigma}^{4} d\left\|P_{t+1}\right\|^{2}\|\widetilde{W}\|^{4}}, \frac{t}{\widehat{\sigma}^{2}\left\|P_{t+1}\right\|\|\widetilde{W}\|^{2}}\right\}\right) \\
& \leq 2 \exp \left(-c \min \left\{\frac{t^{2}}{2 \widehat{\sigma}^{4}\|\widetilde{W}\|^{4} d C^{2}(\boldsymbol{K}) / \underline{\sigma}_{\boldsymbol{X}}{ }^{2}}, \frac{t}{\widehat{\sigma}^{2}\|\widetilde{W}\|^{2} C(\boldsymbol{K}) / \underline{\sigma}_{\boldsymbol{X}}}\right\}\right) .
\end{aligned}
$$

Therefore the random variable $w_{t}^{\top} P_{t+1} w_{t}$ is subexponential with parameters

$$
\left(\frac{\widehat{\sigma}^{4}\|\widetilde{W}\|^{4} d C^{2}(\boldsymbol{K})}{c \underline{\sigma}_{\boldsymbol{X}}{ }^{2}}, \frac{\widehat{\sigma}^{2}\|\widetilde{W}\|^{2} C(\boldsymbol{K})}{2 c \underline{\sigma}_{\boldsymbol{X}}}\right) .
$$

In the same way $x_{0}^{\top} P_{0} x_{0}$ is subexponential with parameters

$$
\left(\frac{\widehat{\sigma}^{4}\left\|\widetilde{W}_{0}\right\|^{4} d C^{2}(\boldsymbol{K})}{c \underline{\sigma}_{\boldsymbol{X}}{ }^{2}}, \frac{\widehat{\sigma}^{2}\left\|\widetilde{W}_{0}\right\|^{2} C(\boldsymbol{K})}{2 c \underline{\sigma}_{\boldsymbol{X}}}\right) .
$$

Let $\bar{\sigma}=\max \left\{\left\|\widetilde{W}_{0}\right\|,\|\widetilde{W}\|\right\}$. Since $\left\{w_{t}\right\}_{t=0}^{T-1}$ are i.i.d. and independent of $x_{0}$, we have that (4.10) is subexponential with parameters

$$
\left((T+1) \frac{\widehat{\sigma}^{4} \bar{\sigma}^{4} d C^{2}(\boldsymbol{K})}{c \underline{\sigma}_{\boldsymbol{X}}^{2}}, \frac{\widehat{\sigma}^{2} \bar{\sigma}^{2} C(\boldsymbol{K})}{2 c \underline{\sigma}_{\boldsymbol{X}}}\right) .
$$

Define

$$
\widetilde{\nabla}_{t}:=\frac{1}{m} \sum_{i=1}^{m}\left(\frac{D}{r^{2}} C\left(\boldsymbol{K}+\boldsymbol{U}_{t}^{i}\right) U_{t}^{i}\right)
$$

as the average of perturbed cost functions across $m$ scenarios, which is an empirical approximation of (4.9). Similarly, define

$$
\widehat{\nabla}_{t}:=\frac{1}{m} \sum_{i=1}^{m}\left(\frac{D}{r^{2}}\left[\sum_{t=0}^{T-1}\left(\left(x_{t}^{i}\right)^{\top} Q_{t} x_{t}^{i}+\left(u_{t}^{i}\right)^{\top} R_{t} u_{t}^{i}\right)+\left(x_{T}^{i}\right)^{\top} Q_{T} x_{T}^{i}\right] U_{t}^{i}\right)
$$

as the average of perturbed and single-trajectory-based cost functions across $m$ scenarios, which is the same as (4.2) in Algorithm 4.1. Note that in order to calculate $\widetilde{\nabla}_{t}$, we require access to $C\left(\boldsymbol{K}+\boldsymbol{U}_{t}^{i}\right)$, which involves the calculation of expectations with respect to unknown initial states and state noises. This may be restrictive in some settings. On the other hand, the calculation of $\widehat{\nabla}_{t}$ only involves single-trajectory-based cost functions.

Lemma 4.11. Assume Assumptions 2.1 and 4.3 hold, and $\underline{\sigma}_{\boldsymbol{X}}>0$. Given any $\epsilon$, there are fixed polynomials $h_{\text {radius }} \in \mathcal{H}(1 / \epsilon, \mathrm{C}(\boldsymbol{K}))$ and $h_{\text {sample }} \in \mathcal{H}(1 / \epsilon, \mathrm{C}(\boldsymbol{K}))$ such

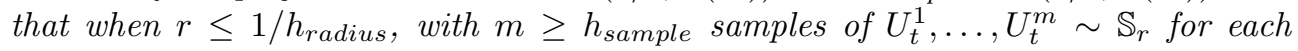
$t=0, \ldots, T-1$,

$$
\left\|\widetilde{\nabla}_{t}-\nabla_{t} C(\boldsymbol{K})\right\|_{F} \leq \epsilon
$$

Copyright (c) by SIAM. Unauthorized reproduction of this article is prohibited. 
holds with high probability (at least $1-\left(\frac{D}{\epsilon}\right)^{-D}$ ). In addition, there is a polynomial $h_{\text {sample }, 2} \in \mathcal{H}(1 / \epsilon, \mathrm{C}(\boldsymbol{K}))$ such that when $r \leq 1 / h_{\text {radius }}$, with $m \geq h_{\text {sample }}+h_{\text {sample }, 2}$ samples of $U_{t}^{1}, \ldots, U_{t}^{m} \sim \mathbb{S}_{r}$ for each $t=0, \ldots, T-1$,

$$
\left\|\widehat{\nabla}_{t}-\nabla_{t} C(\boldsymbol{K})\right\|_{F} \leq \frac{3}{2} \epsilon
$$

holds with high probability (at least $1-2\left(\frac{D}{\epsilon}\right)^{-D}$ ). Here, for each $i=1,2, \ldots, m$, $\left\{x_{t}^{i}\right\}_{t=0}^{T}$ and $\left\{u_{t}^{i}\right\}_{t=0}^{T-1}$ are the dynamics and controls for a single path sampled using policy $\boldsymbol{K}+\boldsymbol{U}_{t}^{i}$.

Proof. Note that

$$
\widetilde{\nabla}_{t}-\nabla_{t} C(\boldsymbol{K})=\left(\nabla_{t} C_{t}^{r}(\boldsymbol{K})-\nabla_{t} C(\boldsymbol{K})\right)+\left(\widetilde{\nabla}_{t}-\nabla_{t} C_{t}^{r}(\boldsymbol{K})\right),
$$

where $C_{t}^{r}$ is defined in (4.8).

For the first term, choose $h_{\text {radius }}=\max \left\{1 / r_{0}, 4 h_{\text {grad }} / \epsilon\right\}$ ( $r_{0}$ is chosen later $)$, where $h_{\text {grad }} \in \mathcal{H}(\mathrm{C}(\boldsymbol{K}))$ is defined as in Lemma 4.8. By Lemma 4.8 when $r \leq$ $1 / h_{\text {radius }} \leq \epsilon / 4 h_{\text {grad }}$, for $\boldsymbol{V}_{t}:=\left(0, \ldots, V_{t}, \ldots, 0\right)$ where $V_{t} \sim \mathbb{B}_{r}$, we have

$$
\left\|\nabla_{t} C\left(\boldsymbol{K}+\boldsymbol{V}_{t}\right)-\nabla_{t} C(\boldsymbol{K})\right\|_{F} \leq h_{\text {grad }}\left\|\boldsymbol{V}_{t}\right\|_{F} \leq h_{\text {grad }} \frac{\epsilon}{4 h_{\text {grad }}}=\frac{\epsilon}{4} .
$$

Since $\nabla_{t} C_{t}^{r}(\boldsymbol{K})=\mathbb{E}_{V_{t} \sim \mathbb{B}_{r}}\left[\nabla_{t} C\left(\boldsymbol{K}+\boldsymbol{V}_{t}\right)\right]$, we have

$$
\left\|\nabla_{t} C\left(\boldsymbol{K}+\boldsymbol{V}_{t}\right)-\nabla_{t} C_{t}^{r}(\boldsymbol{K})\right\|_{F} \leq \frac{\epsilon}{4},
$$

by (4.14) and the continuity of $\nabla_{t} C$. Therefore

$$
\begin{aligned}
\left\|\nabla_{t} C_{t}^{r}(\boldsymbol{K})-\nabla_{t} C(\boldsymbol{K})\right\|_{F} \leq & \left\|\nabla_{t} C\left(\boldsymbol{K}+\boldsymbol{V}_{t}\right)-\nabla_{t} C(\boldsymbol{K})\right\|_{F} \\
& +\left\|\nabla_{t} C\left(\boldsymbol{K}+\boldsymbol{V}_{t}\right)-\nabla_{t} C_{t}^{r}(\boldsymbol{K})\right\|_{F} \leq \frac{\epsilon}{2}
\end{aligned}
$$

holds by triangle inequality. We choose $r_{0}$ such that for any $U_{t} \sim \mathbb{S}_{r}$, we have that $C\left(\boldsymbol{K}+\boldsymbol{U}_{t}\right) \leq 2 C(\boldsymbol{K})$. By Lemma 4.7 , we can pick $1 / r_{0}=h_{\text {cost }} / C(\boldsymbol{K})$; then $\left|C\left(\boldsymbol{K}+\boldsymbol{U}_{t}\right)-C(\bar{K})\right| \leq r_{0} \cdot h_{\text {cost }} \leq C(\boldsymbol{K})$.

For the second term, by Lemma $4.9, \mathbb{E}\left[\widetilde{\nabla}_{t}\right]=\nabla_{t} C_{t}^{r}(\boldsymbol{K})$, and each individual sample is bounded by $2 D C(\boldsymbol{K}) / r$, so by the operator-Bernstein inequality [27, Theorem 12] with

$$
m \geq h_{\text {sample }}=\Theta\left(D\left(\frac{D \cdot C(\boldsymbol{K})}{r \epsilon}\right)^{2} \log (D / \epsilon)\right),
$$

we have

$$
\mathbb{P}\left[\left\|\widetilde{\nabla}_{t}-\nabla_{t} C_{t}^{r}(\boldsymbol{K})\right\|_{F} \leq \frac{\epsilon}{2}\right] \geq 1-\left(\frac{D}{\epsilon}\right)^{-D} .
$$

Note that $h_{\text {sample }} \in \mathcal{H}(1 / \epsilon, \mathrm{C}(\boldsymbol{K}))$ since $1 / r>h_{\text {radius }} \in \mathcal{H}(1 / \epsilon, C(\boldsymbol{K}))$. Adding these two terms together and applying the triangle inequality gives the result.

For the second part, note that

$$
\mathbb{E}_{x_{0}, \boldsymbol{w}}\left[\widehat{\nabla}_{t}\right]=\widetilde{\nabla}_{t}
$$

Copyright $@$ by SIAM. Unauthorized reproduction of this article is prohibited. 
By Lemma 4.10,

$$
\left[\sum_{t=0}^{T-1}\left(\left(x_{t}^{i}\right)^{\top} Q_{t} x_{t}^{i}+\left(u_{t}^{i}\right)^{\top} R_{t} u_{t}^{i}\right)+\left(x_{T}^{i}\right)^{\top} Q_{T} x_{T}^{i}\right]
$$

is subexponential with parameters $\left(\nu^{2}, \alpha\right)$. Therefore,

$$
Z_{i}:=\left(\frac{D}{r^{2}}\left[\sum_{t=0}^{T-1}\left(\left(x_{t}^{i}\right)^{\top} Q_{t} x_{t}^{i}+\left(u_{t}^{i}\right)^{\top} R_{t} u_{t}^{i}\right)+\left(x_{T}^{i}\right)^{\top} Q_{T} x_{T}^{i}\right] U_{t}^{i}\right)
$$

is a subexponential matrix with parameters $\left(\widetilde{\nu}^{2}, \widetilde{\alpha}\right):=\left(\frac{D}{r^{2}} \nu^{2}, \alpha\right)$. Then by the operator-Bernstein inequality [27, Theorem 12],

$$
\mathbb{P}\left[\left\|\frac{1}{m} \sum_{i=1}^{m} Z_{i}-\mathbb{E}\left[Z_{1}\right]\right\|_{F} \leq t\right] \geq 1-2 D \exp \left(-m \frac{t^{2}}{2 \widetilde{\nu}^{2}}\right),
$$

when $t \leq \frac{\widetilde{\nu}^{2}}{\widetilde{\alpha}}$. That is, there exists a polynomial $h_{\text {sample }, 2} \in \mathcal{H}(1 / \epsilon, \mathrm{C}(\boldsymbol{K}))$ where

$$
\begin{aligned}
h_{\text {sample }, 2} & :=h_{\text {sample }, 2}\left(D, \frac{1}{\epsilon}, \frac{1}{r}, \sigma_{0}, \sigma_{w},\left\|\widetilde{W}_{0}\right\|,\|\widetilde{W}\|, C(\boldsymbol{K}), \frac{1}{\underline{\sigma}_{\boldsymbol{X}}}\right) \\
& =\Theta\left(D\left(\frac{\widetilde{\nu}}{\epsilon}\right)^{2} \log (D / \epsilon)\right),
\end{aligned}
$$

such that when $m \geq h_{\text {sample }, 2}$,

$$
\mathbb{P}\left[\left\|\widehat{\nabla}_{t}-\widetilde{\nabla}_{t}\right\|_{F} \leq \frac{\epsilon}{2}\right] \geq 1-\left(\frac{D}{\epsilon}\right)^{-D}
$$

Combining (4.18) with (4.15) and (4.16), we arrive at the desired result.

4.3. Proof of Theorem 4.4. With the results in sections 4.1 and 4.2 , now we are ready to prove the main theorem.

Proof of Theorem 4.4. By Lemma 3.15 and by choosing $\eta \in \mathcal{H}\left(\frac{1}{C\left(\boldsymbol{K}^{0}\right)+1}\right)$ such that the step size condition (3.23) is satisfied,

$$
C\left(\boldsymbol{K}^{\prime}\right)-C\left(\boldsymbol{K}^{*}\right) \leq\left(1-2 \eta \underline{\sigma}_{\boldsymbol{R}} \frac{\underline{\sigma}_{\boldsymbol{X}}^{2}}{\left\|\Sigma_{\boldsymbol{K}^{*}}\right\|}\right)\left(C(\boldsymbol{K})-C\left(\boldsymbol{K}^{*}\right)\right) .
$$

Recall the definition of $\widehat{\nabla}_{t}$ in (4.13), and let $K_{t}^{\prime \prime}=K_{t}-\eta \widehat{\nabla}_{t}$ be the iterate that uses the approximate gradient. We will show later that given enough samples, the gradient can be estimated with enough accuracy to ensure that

$$
\left|C\left(\boldsymbol{K}^{\prime \prime}\right)-C\left(\boldsymbol{K}^{\prime}\right)\right| \leq \eta \underline{\sigma}_{\boldsymbol{R}} \frac{\underline{\sigma}_{\boldsymbol{X}}{ }^{2}}{\| \Sigma_{\boldsymbol{K}^{*} \|}} \epsilon .
$$

This means that as long as $C(\boldsymbol{K})-C\left(\boldsymbol{K}^{*}\right) \geq \epsilon$, we have

$$
C\left(\boldsymbol{K}^{\prime \prime}\right)-C\left(\boldsymbol{K}^{*}\right) \leq\left(1-\eta \underline{\sigma}_{\boldsymbol{R}} \frac{\underline{\sigma}_{\boldsymbol{X}}^{2}}{\left\|\Sigma_{\boldsymbol{K}^{*}}\right\|}\right)\left(C(\boldsymbol{K})-C\left(\boldsymbol{K}^{*}\right)\right) .
$$

Copyright $@$ by SIAM. Unauthorized reproduction of this article is prohibited. 
Then the same proof as that of Theorem 3.3 gives the convergence guarantee.

Now let us prove (4.19). First note that $C\left(\boldsymbol{K}^{\prime \prime}\right)-C\left(\boldsymbol{K}^{\prime}\right)$ is bounded. By Lemma 4.7, if $\left\|K_{t}^{\prime \prime}-K_{t}^{\prime}\right\| \leq \eta \underline{\sigma}_{\boldsymbol{R}} \frac{\underline{\sigma}_{\boldsymbol{X}}{ }^{2}}{\left\|\Sigma_{\boldsymbol{K}^{*}}\right\|} \cdot \epsilon /\left(T \cdot h_{\text {cost }}\right)$, where $h_{\text {cost }} \in \mathcal{H}(\mathrm{C}(\boldsymbol{K}))$ is the polynomial in Lemma 4.7, then (4.19) holds. To get this bound, recall that $K_{t}^{\prime}=K_{t}-\eta \nabla_{t} C(\boldsymbol{K})$ in (3.22), and writing $\nabla_{t}=\nabla_{t} C(\boldsymbol{K})$ for ease of exposition, observe that $K_{t}^{\prime \prime}-K_{t}^{\prime}=$ $\eta\left(\nabla_{t}-\widehat{\nabla}_{t}\right)$; therefore it suffices to ensure that

$$
\left\|\nabla_{t}-\widehat{\nabla}_{t}\right\| \leq \frac{\underline{\sigma}_{\boldsymbol{X}}^{2} \underline{\sigma}_{\boldsymbol{R}}}{T\left\|\Sigma_{\boldsymbol{K}^{*}}\right\| h_{\text {cost }}} \epsilon .
$$

By Lemma 4.11, it is enough to pick

$$
\bar{h}_{\text {radius }}=h_{\text {radius }}\left(3 T\left\|\Sigma_{\boldsymbol{K}^{*}}\right\| h_{\text {cost }}(C(\boldsymbol{K})) /\left(2 \underline{\sigma}_{\boldsymbol{X}}{ }^{2} \underline{\sigma}_{\boldsymbol{R}} \epsilon\right), C(\boldsymbol{K})\right) \in \mathcal{H}(1 / \epsilon, \mathrm{C}(\boldsymbol{K}))
$$

and

$$
\begin{aligned}
\bar{h}_{\text {sample }}= & h_{\text {sample }}\left(\frac{3 h_{\text {cost }}(C(\boldsymbol{K}))\left\|\Sigma_{\boldsymbol{K}^{*}}\right\|}{2 \underline{\sigma}_{\boldsymbol{X}}^{2} \underline{\sigma}_{\boldsymbol{R}} \epsilon}, C(\boldsymbol{K})\right) \\
& +h_{\text {sample }, 2}\left(\frac{3 h_{\text {cost }}(C(\boldsymbol{K}))\left\|\Sigma_{\boldsymbol{K}^{*}}\right\|}{2 \underline{\sigma}_{\boldsymbol{X}}{ }^{2} \underline{\sigma}_{\boldsymbol{R}} \epsilon}, C(\boldsymbol{K})\right) .
\end{aligned}
$$

This gives the desired upper bound on $\left\|\nabla_{t}-\widehat{\nabla}_{t}\right\|$ with high probability (at least $\left.1-2(\epsilon / D)^{D}\right)$.

Since the number of steps is a polynomial, we have $T N=o\left(\epsilon^{D}\right)$. By the union bound with probability at least

$$
\left(1-2\left(\frac{\epsilon}{D}\right)^{D}\right)^{T N} \geq 1-2 T N\left(\frac{\epsilon}{D}\right)^{D} \geq 1-\exp (-D),
$$

we have $\left\|\nabla_{t}-\widehat{\nabla}_{t}\right\| \leq \frac{\sigma_{\boldsymbol{X}}^{2} \underline{\sigma}_{\boldsymbol{R}}}{T\left\|\bar{\Sigma}_{\boldsymbol{K}^{*}}\right\| h_{\text {cost }}} \epsilon t=0,1, \ldots, T-1$. Therefore,

$$
C\left(\boldsymbol{K}^{\prime \prime}\right)-C\left(\boldsymbol{K}^{*}\right) \leq\left(1-\eta \underline{\sigma}_{\boldsymbol{R}} \frac{\underline{\sigma}_{\boldsymbol{X}}{ }^{2}}{\left\|\Sigma_{\boldsymbol{K}^{*}}\right\|}\right)\left(C(\boldsymbol{K})-C\left(\boldsymbol{K}^{*}\right)\right) .
$$

This implies $C\left(\boldsymbol{K}^{\prime \prime}\right)<C(\boldsymbol{K})$. To guarantee that (4.20) holds at each iteration $n=$ $1,2, \ldots, N$, it suffices to pick $\bar{h}_{\text {radius }} \in \mathcal{H}\left(1 / \epsilon, \mathrm{C}\left(\boldsymbol{K}^{0}\right)\right)$ and $\bar{h}_{\text {sample }} \in \mathcal{H}\left(1 / \epsilon, \mathrm{C}\left(\boldsymbol{K}^{0}\right)\right)$. The rest of the proof is the same as that of Theorem 3.3. Note again that in the smoothing, because the function value is monotonically decreasing, and by the choice of radius, all the function values encountered are bounded by $2 C\left(\boldsymbol{K}^{0}\right)$, so the polynomials are indeed bounded throughout the algorithm.

\subsection{Discussion.}

Remark 4.12 (comparison with [22]). The proofs of our main results, Theorems 3.3 and 4.4, are different from those in [22]. First, to prove the gradient dominant condition, [22] only required conditions on the distribution of the initial position. However, we need conditions to guarantee the nondegeneracy of the state covariance matrix at any time. Second, the extra randomness from the sub-Gaussian noise needs to be taken care of in the perturbation analysis of $\Sigma_{\boldsymbol{K}}$. Finally, we need more advanced concentration inequalities to provide the number of samples and the number of simulation trajectories that lead to the theoretical guarantee in the case with unknown parameters. 
Remark 4.13 (nonstationary dynamics). Note that our framework can be generalized to nonstationary dynamics; that is, for $t=0,1, \ldots, T-1$,

$$
x_{t+1}=A_{t} x_{t}+B_{t} u_{t}+w_{t}, x_{0} \sim \mathcal{D},
$$

with $\left\{A_{t}\right\}_{t=0}^{T-1}$ and $\left\{B_{t}\right\}_{t=0}^{T-1}$ time-dependent state parameters.

Remark 4.14 (other policy gradient methods). Our convergence and sample complexity analysis could be applied to other policy gradient methods, including the natural policy gradient method and the Gauss-Newton method, within the framework of the LQR with stochastic dynamics and finite horizons.

5. Numerical experiments. The performance of the PPG algorithm (4.4) is demonstrated for the optimal liquidation problem with a single asset, and the empirical analysis of the policy gradient method (4.1) in higher dimensions is also provided with synthetic data. We will specifically focus on the following questions.

- In practice, how fast do the policy gradient algorithm and the PPG algorithm with known and unknown parameters converge to the true solution?

- How does the deadline (the finite horizon) influence the optimal policy?

- When the real-word system does not exactly follow the LQR framework, does the policy gradient method outperform misspecified LQR models?

This section is organized as follows. We demonstrate the performance of the PPG algorithms for the optimal liquidation problem with a single asset in the LQR framework in section 5.1. We then show that without the LQR model specification, the learned policy from the policy gradient algorithm improves the Almgren-Chriss solution in section 5.2. Finally, we test the performance of the algorithm with unknown parameters in high dimensions in section 5.3.

Note that the policy gradient method outperforms the Q-learning algorithm, a popular model-free method, in terms of both sample complexity and accuracy in our setting. An illustration in a one-dimensional example can be found in [29].

5.1. Optimal liquidation within the LQR framework. Recall the set-up of the optimal liquidation problem in (2.1). By convention, we write the control in the feedback form as $u_{t}=-K_{t} x_{t}$. Writing $K_{t}=\left(k_{t}^{1}, k_{t}^{2}\right)$, we have $u_{t}=-k_{t}^{1} S_{t}-k_{t}^{2} q_{t}$, and the state equation becomes

$$
x_{t+1}=\left(\begin{array}{cc}
1+\gamma k_{t}^{1} & \gamma k_{t}^{2} \\
k_{t}^{1} & 1+k_{t}^{2}
\end{array}\right) x_{t}+w_{t} .
$$

In the liquidation problem, we assume $u_{t} \geq 0(0 \leq t \leq T-1)$. That is, $k_{t}^{1} \leq 0$ and $k_{t}^{2} \leq 0(0 \leq t \leq T-1)$.

Assumption 5.1 (assumptions for the optimal liquidation problems). We assume

(1) $\gamma k_{t}^{1}+k_{t}^{2}>-1(0 \leq t \leq T-1)$;

(2) $\beta>\frac{\gamma}{2}$.

Justification of the assumption. Assumption 5.1(1) is essential to ensure that the liquidation problem is well-defined. First, $\gamma k_{t}^{1}>-1$ makes sure that the stock price process $\left\{S_{t}\right\}_{t=0}^{T}$ is well-behaved:

$$
\mathbb{E}\left[S_{t+1}\right]=\mathbb{E}\left[S_{t}\right]-\gamma \mathbb{E}\left[u_{t}\right]=\left(1+\gamma k_{t}^{1}\right) \mathbb{E}\left[S_{t}\right]+\gamma k_{t}^{2} q_{t} .
$$

If $\gamma k_{t}^{1}<-1$, then $\mathbb{E}\left[S_{t+1}\right] \leq 0$ since $k_{t}^{2} \leq 0$. Second, $k_{t}^{2} \geq-1$ guarantees that inventory will not be negative. Note that

$$
q_{t+1}=q_{t}-\left(-k_{t}^{1} S_{t}-k_{t}^{2} q_{t}\right)=\left(1+k_{t}^{2}\right) q_{t}+k_{t}^{1} S_{t} .
$$


If $k_{t}^{2} \leq-1$ and $q_{t}>0$, then $q_{t+1}<0$. Assumption 5.1(2) implies that the temporary market impact is "bigger" than one-half of the permanent market impact, which is consistent with the empirical evidence [8] and assumptions in [7].

Learning to liquidate. In practice, traders may not know the market impact parameter $\gamma$. But one can always take some $\bar{\gamma}>\gamma$ based on some basic understandings of the market and perform a PPG algorithm to the closed convex set $\mathcal{S}$ :

$$
\begin{aligned}
\mathcal{S}:=\left\{\boldsymbol{K}=\left(K_{0}, \ldots, K_{T-1}\right): K_{t}=\left(k_{t}^{1}, k_{t}^{2}\right), \bar{\gamma} k_{t}^{1}+k_{t}^{2} \geq-1+\zeta, k_{t}^{1} \leq 0, k_{t}^{2} \leq 0\right. \\
\forall t=0, \ldots, T-1\},
\end{aligned}
$$

with some small parameter $\zeta>0$.

In practice $\gamma$ is usually on the order of $10^{-5} \sim 10^{-6}$ (see details in [29]), and hence a universal upper bound $\bar{\gamma}$ in (5.1) is not a strong assumption for liquidating a given portfolio of stocks.

Proposition 5.2. Assume $\boldsymbol{K} \in \mathcal{S}$ and Assumptions 2.1, 4.3, and 5.1 hold; we have that $\underline{\sigma}_{\boldsymbol{X}}>0$ and that $\left\{P_{t}^{K}\right\}_{t=0}^{T}$ derived from (3.9) are positive definite for the optimal liquidation problem (2.7) and (2.9).

The proof of Proposition 5.2 is deferred to [29]. It is easy to check that the projection set $\mathcal{S}$ defined in (5.1) is convex and closed. Along with Proposition 5.2, the convergence result in Theorem 4.5 holds for the liquidation problem (2.7) and (2.9) as long as the conditions in Proposition 5.2 are satisfied.

We test the performance of the PPG algorithm with projection set $\mathcal{S}$ on Apple (AAPL) and Facebook (FB) stocks. The market simulator of the associated LQR framework is constructed with NASDAQ ITCH data, and the details can be found in [29].

Performance measure. We use the following normalized error to quantify the performance of a given policy $K$ :

$$
\text { Normalized error }=\frac{C(\boldsymbol{K})-C\left(\boldsymbol{K}^{*}\right)}{C\left(\boldsymbol{K}^{*}\right)},
$$

where $\boldsymbol{K}^{*}$ is the optimal policy defined in (2.5).

Set-up. (1) Parameters: $\phi=5 \times 10^{-6}$ (for both AAPL and FB), $\epsilon=10^{-8}$, $T=10$; smoothing parameter $r=0.6$, number of trajectories $m=200$; initial policy $\boldsymbol{K}^{0} \in \mathbb{R}^{1 \times 2 T}$ with $\left\{\boldsymbol{K}^{0}\right\}_{i j}=-0.2$ for all $i, j$, for both algorithms with known and unknown parameters; step sizes as indicated in the figures; $\bar{\gamma}=5 \times 10^{-5}, \zeta=10^{-12}$ for the projection set. (2) Initialization: assume the initial inventory $q_{0}$ follows $\mathcal{N}(500,1)$. The small variance of the initial inventory distribution is used to guarantee the initial state covariance matrix is positive definite. In practice, the algorithm converges with deterministic initial inventories.

Convergence. PPG algorithms with both known parameters and unknown parameters show a reasonable level of accuracy within 50 iterations (that is, the normalized error is less than $10^{-2}$ ). The PPG algorithm with known parameters has almost no fluctuations across the 50 scenarios. By choosing $m=200$, the performance of the PPG algorithm with unknown parameters is stable with relatively small fluctuations (see the shaded area in Figure 1b) across the 50 scenarios.

Impact of the deadline. The optimal policy is sensitive to the deadline in that the shapes of the optimal inventory trajectories are different with different deadlines. See 


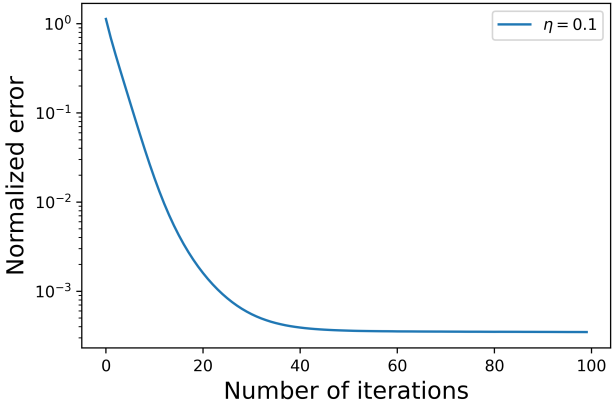

(a) PPG with known parameters $(\eta=0.1)$.

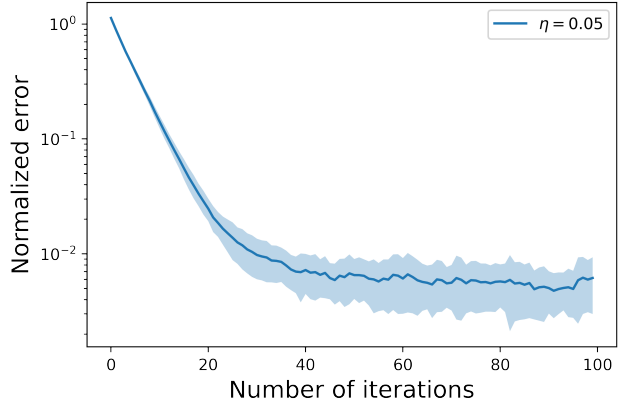

(b) PPG with unknown parameters $(\eta=$ $0.05)$.

FiG. 1. Performance of the PPG algorithms (50 simulation scenarios).

Figure 2 for both AAPL and FB with $T=30,60$, and 120 minutes. The liquidation speed is almost linear when $T$ is small, and it is faster in the initial trading phase and slower at the end when $T$ is relatively large.

Impact of the parameter $\phi$. Recall that in (2.9) the parameter $\phi$ is used to balance the expected terminal wealth $\mathbb{E}[C]$ and the variance of the terminal wealth $\operatorname{var}[C]$. To show the impact of $\phi$, we set $\phi$ to be $10^{-4}, 10^{-5}, 10^{-6}$, and $10^{-7}$ and show the corresponding inventory trajectories in Figure 3. The optimal liquidation speed is almost linear when $\phi$ is small, while it is faster in the initial trading phase and slower at the end when $\phi$ is relatively large.

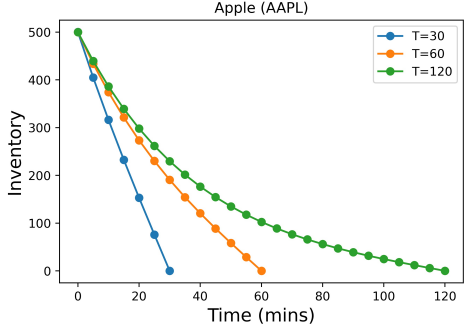

(a) AAPL

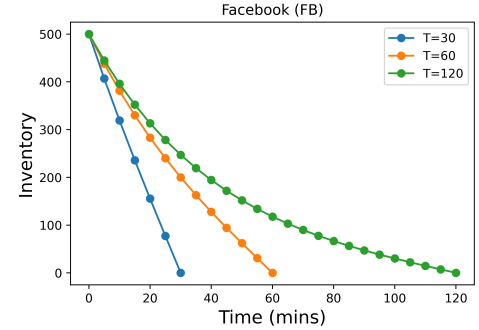

(b) FB.

FIG. 2. Optimal inventory trajectory under different deadlines (200 simulation scenarios).

Impact of the parameter $\epsilon$. Recall that our liquidation formulation (2.9) differs from the Almgren-Chriss formulation (2.8) by an additional regularization term $\sum_{t=0}^{T} \epsilon S_{t}^{2}$. The role of this term is to enable the problem to be cast in the LQR framework and to guarantee the well-definedness of the Ricatti equation. From Figure 4a, the optimal policies and inventory trajectories are close to those of the AlmgrenChriss solution when $\epsilon \leq 0.01$. However, when $\epsilon=0.05$, the optimal policy is far away from the Almgren-Chriss solution. We show the difference between $C_{\mathrm{AC}}$, defined in $(2.8)$, and $C_{\mathrm{LQR}}(\epsilon)$, defined in (2.9), in Figure $4 \mathrm{~b}$. We see that $C_{\mathrm{LQR}}(\epsilon)$ is close to $C_{\mathrm{AC}}$ when $\epsilon<0.02$ and is markedly different from $C_{\mathrm{AC}}$ when $\epsilon \geq 0.02$. It is worth noticing that when $\epsilon=0$, the algorithm does converge to the Almgren-Chriss solution in our setting although the convergence of the algorithm in this case is not 


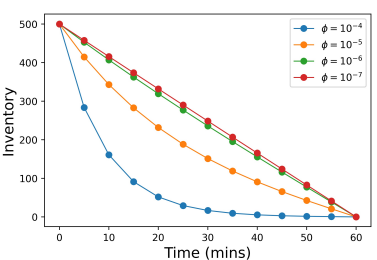

FIG. 3. Inventory trajectories of $A A P L$ under different $\phi$ (average across 200 simulation scenarios).

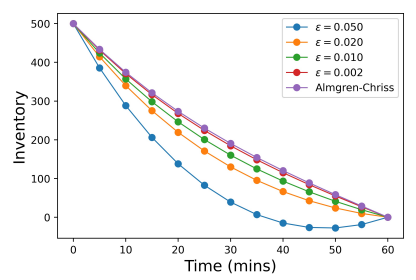

(a) Inventory trajectories.

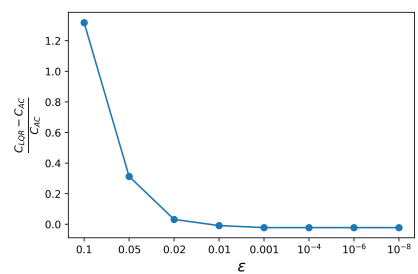

(b) Relative cost difference.

FIG. 4. Original Almgren-Chriss framework versus LQR formulation under different $\epsilon$ (AAPL).

guaranteed by our theoretical results.

5.2. Learning to liquidate without model specification. In practice, the dynamics of the trading system may not be exactly those assumed in the LQR framework but we might expect that the policy gradient method could still perform well when the system is "nearly" linear quadratic as the execution of the policy gradient method does not rely on the model specification. In this section, we consider liquidation problems in the Limit Order Book (LOB) setting. An LOB is a list of orders that a trading venue, for example, the NASDAQ exchange, uses to record the interest of buyers and sellers in a particular financial instrument. There are two types of orders the buyers (sellers) can submit: a limit buy (sell) order with a preferred price for a given volume, or a market buy (sell) order with a given volume which will be immediately executed with the best available limit sell (buy) orders. Here we perform the policy gradient method to learn the optimal strategies to liquidate using market orders in the LOB.

We denote by $S_{t}$ the mid-price of the asset at time $t$, that is, the average of the best-bid price and best-ask price. At each time $t$, the decision is to liquidate an amount $u_{t}$ of the asset. The action $u_{t}$ will have an impact on the market, with possibly both temporary and permanent impacts. Unlike the LQR framework or the classical Almgren-Chriss model, where dynamics are assumed to follow some stochastic model, here we run the policy gradient method directly on the LOB without any assumption on how the mid-price $S_{t}$ moves and what the forms of the market impacts are. Denote by $q_{t}=q_{t-1}-u_{t-1}$ the inventory at time $t$. We restrict the admissible controls to be of the linear feedback form $u_{t}=-K_{t}\left(S_{t}, q_{t}\right)^{\top}$ with some $K_{t} \in \mathbb{R}^{1 \times 2}$.

The cost $c_{t}=\phi^{\prime}\left(q_{t}-u_{t}\right)^{2}-r_{t}\left(u_{t}\right)$ at time $t$ consists of two parts. The first part $\phi^{\prime}\left(q_{t}-u_{t}\right)^{2}$ is the holding cost of the inventory weighted by a parameter $\phi^{\prime}$. The quantity $r_{t}\left(u_{t}\right)$ is the amount we receive by liquidating $u_{t}$ shares at time $t$. Note that $r_{t}(\cdot)$ may depend on $S_{t}$ and other market observables. For example, if we liquidate $u_{t}=1000$ shares of the asset with the market conditions given in Table 1, then the amount received would be

$$
r_{t}\left(u_{t}\right)=397 \times 200.1+412 \times 200.0+(1000-397-412) \times 199.9=200020.6 .
$$

This transaction moves the best bid price two levels down. This is commonly referred to as the temporary impact of a market order.

Performance metric: implementation shortfall [41].

$$
\operatorname{IS}(\boldsymbol{u})=\left(\sum_{t=0}^{T-1} c_{t}\left(u_{t}\right)+c_{T}\left(q_{0}-\sum_{t=0}^{T-1} u_{t}\right)\right)-c_{0}\left(q_{0}\right) .
$$

Copyright $@$ by SIAM. Unauthorized reproduction of this article is prohibited. 
TABLE 1

One snapshot of the $L O B$.

\begin{tabular}{lccccc}
\hline Bid level & One & Two & Three & Four & Five \\
\hline \hline Bid price (USD) & 200.1 & 200.0 & 199.9 & 199.8 & 199.7 \\
\hline Volume available & 397 & 412 & 502 & 442 & 529 \\
\hline
\end{tabular}

The first term of (5.2) is the cost of implementing policy $\boldsymbol{u}$ over the horizon $[0, T]$. The second term is the cost when liquidating $q_{0}$ market orders at time 0 . If we expect $\boldsymbol{u}$ is better than liquidating everything at time 0 , then $\operatorname{IS}(\boldsymbol{u})<0$. A smaller implementation shortfall implies that the strategy is more profitable.

We use the following relative performance (evaluated on a single trajectory) to compare the performance of two policies $\boldsymbol{u}^{1}$ and $\boldsymbol{u}^{2}$ :

$$
\text { Relative performance }=\frac{\operatorname{IS}\left(\boldsymbol{u}^{2}\right)-\operatorname{IS}\left(\boldsymbol{u}^{1}\right)}{\left|\operatorname{IS}\left(\boldsymbol{u}^{2}\right)\right|} .
$$

Experiment set-up. We consider the LOB data consisting of the best five levels, and we assume that the trading frequency $\Delta=1$ minute and the trading horizon $T=10$ minutes. We perform a numerical analysis for five different stocks, Apple (AAPL), Facebook (FB), International Business Machines Corporation (IBM), American Airlines (AAL), and JP Morgan (JPM), during the period from 01/01/2019 to $12 / 31 / 2019$. The data is divided into two sets, a training set with data between 10:00AM-12:00AM 01/01/2019-08/31/2019 and a test set with data between 10:00AM-12:00AM 09/01/2019-12/31/2019.

We take $\phi^{\prime}=5 \times 10^{-6} ; T=10$; smoothing parameter $r=0.4$; number of trajectories $m=200$; initial policy $\boldsymbol{K}^{0} \in \mathbb{R}^{1 \times 20}$ with $\left(\boldsymbol{K}^{0}\right)_{i j}=-0.2$ for all $i, j$; and step size $\eta=10^{-6}$. We assume the initial inventory follows $q_{0}=2000$. We compare the performance of the policy gradient method with the Almgren-Chriss solution with fitted parameters given in Table 3 in [29]. In the Almgren-Chriss model, we set $\phi=\sigma^{2} \phi^{\prime}$ to ensure a reasonable comparison.

Results. From Table 2 and Figure 5, the policy gradient method improves on the Almgren-Chriss solution by around $20 \%$ on five different stocks from different financial sectors. Note that the goal of the policy gradient method is to learn the global minimizer of the expected cost function; hence it is expected that the AlmgrenChriss solution could perform better than the policy gradient method for some sample trajectories, as shown in Figure 5. This result is compatible with the performance of the Q-learning algorithms [30]. The drawback of Q-learning algorithms is that the computational complexity is highly dependent on the size of the set of (discrete) states and actions, whereas the policy gradient method can handle continuous states and actions.

We conjecture that the policy gradient method may be capable of learning the global "optimal" solution for a larger class of models that are "similar" to the LQR framework with stochastic dynamics and finite time horizon. In addition, as the policy gradient method is a model-free algorithm, it is more robust with respect to model misspecification as compared to the Almgren-Chriss framework.

5.3. Learning $\mathbf{L Q R}$ in higher dimensions. In practice we can perform the policy gradient method for the optimal liquidation problem with multiple assets. However, it is difficult to capture the cross impact and permanent impact with historical LOB data. Therefore we test the performance of the policy gradient method in higher 
TABLE 2

Average relative performance of the policy gradient $\left(\boldsymbol{u}^{1}\right)$ compared to the Almgren-Chriss solution $\left(\boldsymbol{u}^{2}\right)$.

\begin{tabular}{lrrrrr}
\hline Asset & IBM & AAL & JPM & FB & AAPL \\
\hline \hline In sample & 0.173 & 0.152 & 0.251 & 0.181 & 0.165 \\
(std) & $(0.09)$ & $(0.27)$ & $(0.31)$ & $(0.32)$ & $(0.31)$ \\
\hline Out of sample & 0.178 & 0.146 & 0.245 & 0.175 & 0.163 \\
(std) & $(0.08)$ & $(0.29)$ & $(0.36)$ & $(0.24)$ & $(0.37)$ \\
\hline
\end{tabular}

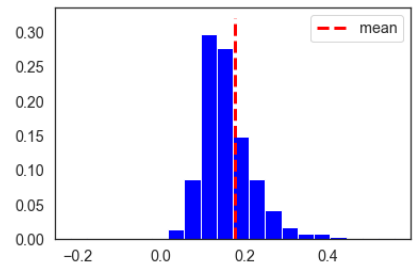

(a) IBM.

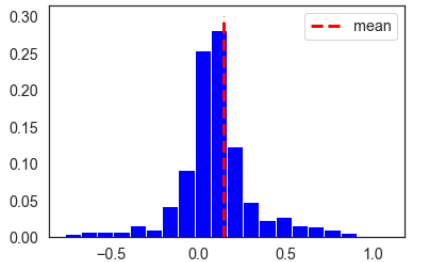

(b) AAL.

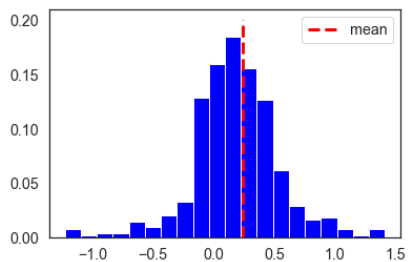

(c) JPM.

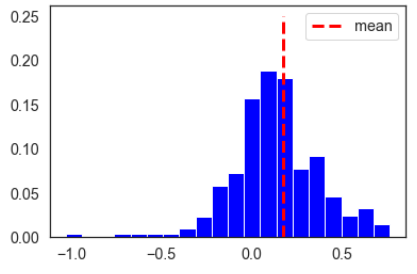

(d) FB.

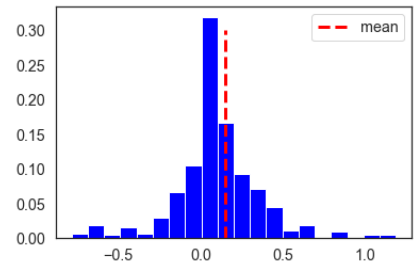

(e) AAPL.

FIG. 5. Empirical distribution of the relative performance on the test set.

dimensions on synthetic data consisting of a four-dimensional state variable and a two-dimensional control variable. The parameters are randomly picked such that the conditions for our LQR framework are satisfied.

Set-up. (1) Parameters:

$$
\begin{gathered}
A=\left(\begin{array}{cccc}
0.5 & 0.05 & 0.1 & 0.2 \\
0 & 0.2 & 0.3 & 0.1 \\
0.06 & 0.1 & 0.2 & 0.4 \\
0.05 & 0.2 & 0.15 & 0.1
\end{array}\right), \quad B=\left(\begin{array}{cc}
-0.05 & -0.01 \\
-0.005 & -0.01 \\
-1 & -0.01 \\
-0.01 & -0.9
\end{array}\right), \\
Q_{t}=\left(\begin{array}{cccc}
1 & 0.2 & -0.005 & 0.015 \\
0.2 & 1.1 & 0.15 & 0 \\
-0.05 & 0.15 & 0.9 & -0.08 \\
0.015 & 0 & -0.08 & 0.88
\end{array}\right), \quad R_{t}=\left(\begin{array}{cc}
0.4 & -0.25 \\
-0.25 & 0.7
\end{array}\right), \\
W=\left(\begin{array}{cccc}
0.1 & 0 & 0 & 0 \\
0 & 0.5 & 0 & 0 \\
0 & 0 & 0.2 & 0 \\
0 & 0 & 0 & 0.3
\end{array}\right),
\end{gathered}
$$

Copyright $@$ ㅇ by SIAM. Unauthorized reproduction of this article is prohibited. 
$Q_{T}=Q_{t}, T=10$; smoothing parameter $r=1$, number of trajectories $m=200$; initial policy $\boldsymbol{K}^{0} \in \mathbb{R}^{2 \times 40}$ with $\left\{\boldsymbol{K}^{0}\right\}_{i j}=0.05$ for all $i, j$, for both known and unknown parameters.

(2) Initialization: We assume that $x_{0}=\left(x_{0}^{1}, x_{0}^{2}, x_{0}^{3}, x_{0}^{4}\right)^{\top}$ and $x_{0}^{i}$ are independent. $x_{0}^{1}, x_{0}^{2}, x_{0}^{3}$, and $x_{0}^{4}$ are sampled from $\mathcal{N}(5,0.1), \mathcal{N}(2,0.3), \mathcal{N}(8,1), \mathcal{N}(5,0.5)$.

Convergence. For the high-dimensional case, the normalized error falls below the threshold $10^{-2}$ within 80 iterations for the policy gradient algorithm with known parameters. It takes substantially more iterations for the policy gradient algorithm with unknown parameters to have an error near such a threshold, which is as expected. See Figure 6.

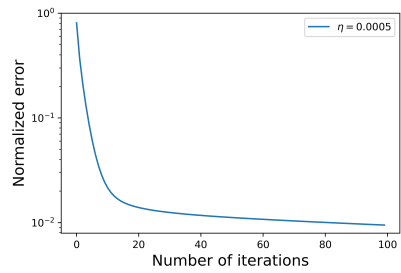

(a) Known parameters $(\eta=0.0005)$.

FIG. 6. Performance of the policy gradient algorithms (50 simulation scenarios).

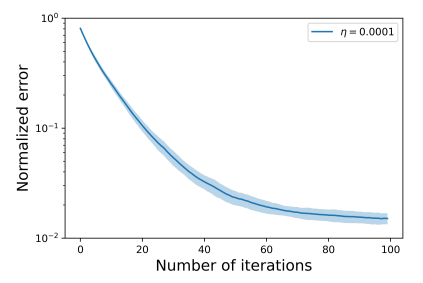

(b) Unknown parameters $(\eta=0.0001)$.

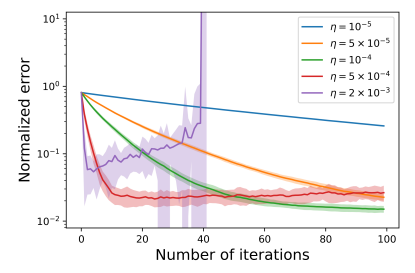

FIG. 7. Performance of the policy gradient algorithm with unknown parameters under different step size $\eta(50$ simulation scenarios). (Color available online.)

Outcomes from varying the parameter $\eta$. The performance of the policy gradient algorithm also depends on the values of the step size $\eta$. We show how the values of the step size $\eta \in\left[10^{-5}, 2 \times 10^{-3}\right]$ affect the convergence of the policy gradient algorithm with unknown parameters in Figure 7. A tiny step size leads to slow convergence (see the blue line when $\eta=10^{-5}$ ), and a larger step size may cause divergence (see the purple line when $\eta=2 \times 10^{-3}$ ).

\section{REFERENCES}

[1] Y. Abbasi-Yadkori And C. Szepesvári, Regret bounds for the adaptive control of linear quadratic systems, in Proceedings of the 24th Annual Conference on Learning Theory, 2011, pp. $1-26$.

[2] M. Abeille And A. LazARIC, Thompson sampling for linear-quadratic control problems, in AISTATS 2017 - 20th International Conference on Artificial Intelligence and Statistics, 2017, pp. 1246-1254.

[3] M. Abeille, E. Sérié, A. Lazaric, and X. Brokmann, LQG for Portfolio Optimization, 2016; available at SSRN 2863925, https://ssrn.com/abstract $=2863925$.

[4] R. Adamczak, A note on the Hanson-Wright inequality for random vectors with dependencies, Electron. Commun. Probab., 20 (2015), 72.

[5] A. Alfonsi, A. Fruth, And A. Schied, Optimal execution strategies in limit order books with general shape functions, Quant. Finance, 10 (2010), pp. 143-157.

[6] R. Almgren, Optimal execution with nonlinear impact functions and trading-enhanced risk, Appl. Math. Finance, 10 (2003), pp. 1-18.

[7] R. Almgren and N. Chriss, Optimal execution of portfolio transactions, J. Risk, 3 (2001), pp. $5-40$.

[8] R. Almgren, C. Thum, E. Hauptmann, and H. Li, Direct estimation of equity market impact, Risk, 18 (2005), pp. 58-62.

[9] B. D. O. Anderson And J. B. Moore, Optimal Control: Linear Quadratic Methods, Courier Corporation, 2007.

[10] K. J. Åström and B. Wittenmark, Adaptive Control, Courier Corporation, 2013. 
[11] W. BaO And X.-Y. LiU, Multi-Agent Deep Reinforcement Learning for Liquidation Strategy Analysis, preprint, https://arxiv.org/abs/1906.11046, 2019.

[12] D. Bertsekas, Dynamic Programming and Optimal Control, Vol. 1, 3rd ed., Athena Scientific, 2005, http://gen.lib.rus.ec/book/index.php?md5=f28152a94f3313576017f55b6bb9ffe8.

[13] J. Bhandari and D. Russo, Global Optimality Guarantees for Policy Gradient Methods, preprint, https://arxiv.org/abs/1906.01786, 2019.

[14] J. Bu, A. Mesbahi, M. Fazel, And M. Mesbahi, LQR through the Lens of First Order Methods: Discrete-time Case, preprint, https://arxiv.org/abs/1907.08921, 2019.

[15] J. Bu, A. Mesbahi, And M. Mesbahi, Policy Gradient-based Algorithms for Continuous-time Linear Quadratic Control, preprint, https://arxiv.org/abs/2006.09178, 2020.

[16] J. Bu, L. J. Ratliff, And M. Mesbahi, Global Convergence of Policy Gradient for Sequential Zero-Sum Linear Quadratic Dynamic Games, preprint, https://arxiv.org/abs/1911.04672, 2019.

[17] R. Carmona, M. Laurière, and Z. Tan, Linear-Quadratic Mean-Field Reinforcement Learning: Convergence of Policy Gradient Methods, preprint, https://arxiv.org/abs/1910.04295, 2019.

[18] A. Charpentier, R. Elie, and C. Remlinger, Reinforcement Learning in Economics and Finance, preprint, https://arxiv.org/abs/2003.10014, 2020.

[19] S. Dean, H. Mania, N. Matni, B. Recht, and S. Tu, On the sample complexity of the linear quadratic regulator, Found. Comput. Math., 20 (2020), pp. 633-679.

[20] M. K. S. Faradonbeh, A. Tewari, and G. Michailidis, Optimism-based adaptive regulation of linear-quadratic systems, IEEE Trans. Automat. Control, 66 (2021), pp. 1802-1808.

[21] S. Fattahi, N. Matni, and S. Sojoudi, Efficient learning of distributed linear-quadratic control policies, SIAM J. Control Optim., 58 (2020), pp. 2927-2951, https://doi.org/10.1137/ 19M1291108.

[22] M. Fazel, R. Ge, S. M. Kakade, and M. Mesbahi, Global convergence of policy gradient methods for the linear quadratic regulator, in Proceedings of the 35th International Conference on Machine Learning, 2018, pp. 1467-1476.

[23] C.-N. Fiechter, PAC adaptive control of linear systems, in Proceedings of the Tenth Annual Conference on Computational Learning Theory, 1997, pp. 72-80.

[24] A. D. Flaxman, A. T. Kalai, and H. B. McMahan, Online convex optimization in the bandit setting: Gradient descent without a gradient, in Proceedings of the Sixteenth Annual ACMSIAM Symposium on Discrete Algorithms, ACM, New York, SIAM, Philadelphia, 2005, pp. 385-394.

[25] J. Gatheral And A. Schied, Optimal trade execution under geometric Brownian motion in the Almgren and Chriss framework, Int. J. Theor. Appl. Finance, 14 (2011), pp. 353-368.

[26] B. Gravell, P. M. Esfahani, and T. Summers, Learning Robust Controllers for Linear Quadratic Systems with Multiplicative Noise via Policy Gradient, preprint, https://arxiv. org/abs/1905.13547, 2019.

[27] D. Gross, Recovering low-rank matrices from few coefficients in any basis, IEEE Trans. Inform. Theory, 57 (2011), pp. 1548-1566.

[28] X. Guo, R. Xu, and T. Zariphopoulou, Entropy Regularization for Mean Field Games with Learning, preprint, https://arxiv.org/abs/2010.00145, 2020.

[29] B. Hambly, R. Xu, And H. Yang, Policy Gradient Methods for the Noisy Linear Quadratic Regulator over a Finite Horizon, preprint, https://arxiv.org/pdf/2011.10300.pdf, 2021.

[30] D. Hendricks and D. Wilcox, A reinforcement learning extension to the Almgren-Chriss framework for optimal trade execution, in 2014 IEEE Conference on Computational Intelligence for Financial Engineering \& Economics (CIFEr), IEEE, 2014, pp. 457-464.

[31] M. Ibrahimi, A. Javanmard, and B. V. Roy, Efficient reinforcement learning for high dimensional linear quadratic systems, in Advances in Neural Information Processing Systems, 2012, pp. 2636-2644.

[32] Z. Jin, J. M. Schmitt, And Z. Wen, On the Analysis of Model-free Methods for the Linear Quadratic Regulator, preprint, https://arxiv.org/abs/2007.03861, 2020.

[33] L. Leal, M. Laurière, AND C.-A. Lehalle, Learning a Functional Control for High-Frequency Finance, preprint, https://arxiv.org/abs/2006.09611, 2020.

[34] W. Li AND E. Todorov, Iterative linear quadratic regulator design for nonlinear biological movement systems, in International Conference on Informatics in Control, Automation and Robotics (ICINCO), 2004, pp. 222-229.

[35] D. Malik, A. Pananjady, K. Bhatia, K. Khamaru, P. Bartlett, and M. Wainwright, Derivative-free methods for policy optimization: Guarantees for linear quadratic systems, J. Mach. Learn. Res., 21 (2020), 21.

[36] Y. Nesterov, Introductory Lectures on Convex Optimization: A Basic Course, Appl. Optim. 
87, Springer Science \& Business Media, 2003.

[37] Y. Nevmyvaka, Y. Feng, and M. Kearns, Reinforcement learning for optimized trade execution, in Proceedings of the 23rd International Conference on Machine Learning, 2006, pp. 673-680.

[38] B. Ning, F. H. T. Ling, and S. Jaimungal, Double Deep Q-Learning for Optimal Execution, preprint, https://arxiv.org/abs/1812.06600, 2018.

[39] Y. Ouyang, M. Gagrani, and R. Jain, Control of unknown linear systems with Thompson sampling, in 2017 55th Annual Allerton Conference on Communication, Control, and Computing (Allerton), IEEE, 2017, pp. 1198-1205.

[40] P. Patrinos, S. Trimboli, And A. Bemporad, Stochastic MPC for real-time market-based optimal power dispatch, in 2011 50th IEEE Conference on Decision and Control and European Control Conference, IEEE, 2011, pp. 7111-7116.

[41] A. F. Perold, The implementation shortfall: Paper versus reality, J. Portfolio Management, 14 (1988), pp. 4-9.

[42] B. Recht, A tour of reinforcement learning: The view from continuous control, Annu. Rev. Control Robotics Autonomous Systems, 2 (2019), pp. 253-279, https://doi.org/10.1146/ annurev-control-053018-023825.

[43] S. Tu AND B. ReCht, Least-squares temporal difference learning for the linear quadratic regulator, in International Conference on Machine Learning, 2018, pp. 5005-5014.

[44] S. TU AND B. RECHT, The gap between model-based and model-free methods on the linear quadratic regulator: An asymptotic viewpoint, in Conference on Learning Theory, 2019, pp. 3036-3083.

[45] Y. Wasa, K. Sakata, K. Hirata, and K. Uchida, Differential game-based load frequency control for power networks and its integration with electricity market mechanisms, in 2017 IEEE Conference on Control Technology and Applications (CCTA), IEEE, 2017, pp. 10441049.

[46] Z. Yang, Y. Chen, M. Hong, and Z. Wang, On the Global Convergence of Actor-Critic: A Case for Linear Quadratic Regulator with Ergodic Cost, preprint, https://arxiv.org/abs/ 1907.06246, 2019.

[47] K. Zhang, Z. Yang, and T. Basar, Policy optimization provably converges to Nash equilibria in zero-sum linear quadratic games, in Advances in Neural Information Processing Systems, 2019, pp. 11602-11614.

[48] Z. Zhang, S. Zohren, And S. Roberts, Deep reinforcement learning for trading, J. Financial Data Sci., 2 (2020), pp. 25-40.

Copyright (c) by SIAM. Unauthorized reproduction of this article is prohibited. 